\title{
The Impact of Operational Decisions on the Design of Salesforce Incentives
}

\author{
Soroush Saghafian, ${ }^{1}$ Xiuli Chao ${ }^{2}$ \\ ${ }^{1}$ Industrial Engineering, School of Computing, Informatics and Decision Systems Engineering, \\ Arizona State University, Tempe, Arizona \\ ${ }^{2}$ Department of Industrial and Operations Engineering, University of Michigan, Ann Arbor, Michigan
}

Received 21 June 2013; accepted 27 March 2014

DOI 10.1002/nav.21585

Published online 8 May 2014 in Wiley Online Library (wileyonlinelibrary.com).

\begin{abstract}
When facing high levels of overstock inventories, firms often push their salesforce to work harder than usual to attract more demand, and one way to achieve that is to offer attractive incentives. However, most research on the optimal design of salesforce incentives ignores this dependency and assumes that operational decisions of production/inventory management are separable from design of salesforce incentives. We investigate this dependency in the problem of joint salesforce incentive design and inventory/production control. We develop a dynamic Principal-Agent model with both Moral Hazard and Adverse Selection in which the principal is strategic and risk-neutral but the agent is myopic and risk-averse. We find the optimal joint incentive design and inventory control strategy, and demonstrate the impact of operational decisions on the design of a compensation package. The optimal strategy is characterized by a menu of inventory-dependent salesforce compensation contracts. We show that the optimal compensation package depends highly on the operational decisions; when inventory levels are high, (a) the firm offers a more attractive contract and (b) the contract is effective in inducing the salesforce to work harder than usual. In contrast, when inventory levels are low, the firm can offer a less attractive compensation package, but still expect the salesforce to work hard enough. In addition, we show that although the inventory/production management and the design of salesforce compensation package are highly correlated, information acquisition through contract design allows the firm to implement traditional inventory control policies: a market-based state-dependent policy (with a constant base-stock level when the inventory is low) that makes use of the extracted market condition from the agent is optimal. This work appears to be the first article on operations that addresses the important interplay between inventory/production control and salesforce compensation decisions in a dynamic setting. Our findings shed light on the effective integration of these two significant aspects for the successful operation of a firm. (c) 2014 Wiley Periodicals, Inc. Naval Research Logistics 61: 320-340, 2014
\end{abstract}

Keywords: dynamic incentive design; principal agent; moral hazard; adverse selection; inventory control

\section{INTRODUCTION}

Marketing and Operations Management (OM) represent two important sections of most firms. Within a company, marketing personnel try to increase demand and operations personnel try to effectively match supply with the attracted demand. Both of these two sections' activities also represent considerable investments for a firm. For instance, while operational activities (e.g., logistics, warehousing, production/inventory, etc.) are widely known to be a major cost for a firm, its salesforce may cost 5-40\% of total sales ([47]).

Because of their importance, a vast stream of research has been devoted to developing insights into improving the marketing and operational activities. However, the literature on effective dynamic mechanisms to integrate these activities

Correspondence to: Soroush Saghafian (soroush.saghafian@ asu.edu) is still immature and growing (see e.g., [37, 25]). The importance of filling this gap and the need for research on integrating these important activities have been discussed in several studies including the Harvard Business Review article titled "Can marketing and manufacturing coexist?" [44]. Such studies (see also [25]) specifically illuminate the importance of considering marketing decisions when controlling production/inventory activities, and vice versa.

In this article, we consider the joint problem of (a) designing appropriate inventory-dependent contracts to induce the salesforce to attract sufficient demand (marketing) and (b) controlling production/inventory to generate enough supply (operations). In the next section, we will review the literature on both problems, especially the related work from the Marketing literature. Most of the research in this area, however, does not consider the interdependence between salesforce incentive design and production/inventory control. Hence, the incentive design problems studied in the literature 
are typically separated from such operational decisions, and consequently, lacking any coordination, the optimal contract design and the optimal production/inventory strategy become independent decisions. In practice, however, salesforce compensation plans may highly depend on such operational decisions. For instance, when firms face high inventory levels (e.g., as a result of a higher-than-required production or lower-than-expected sales in previous periods), they push their workforces to work harder than usual to enhance customer demand, and offering the salesforce a more attractive compensation plan is a viable and often utilized option. By contrast, when firms are understocked, they are less interested in boosting demand. Hence, they offer a less attractive compensation package as there is no need for a significant effort from the salesforce. Indeed, altering the compensation plans has been empirically found to be an effective method to increase profits through increasing sales, and thereby decreasing the consequences of keeping high levels of unsold inventories (see e.g., [31]). We further note that contract design is a realistic approach used in practice for effective salesforce compensation (see e.g., [21] for a menu of incentive contracts used by IBM).

The problem we consider in this article is faced by many firms. An example is LKQ Corporation that provides a variety of products to collision repair shops as well as mechanical repair shops. The firm needs to effectively control the inventory of its items, which includes new products produced by original equipment manufacturing (OEM), products produced by other companies rather than OEMs (aftermarket products), and recycled and refurbished items. Similar to the assumption we make in this article, LKQ faces nonstationary and seasonal demand: "During the winter months, we tend to have higher demand for our products because there are more weather related accidents, which generate repair" ([13]). Moreover, as is our focus in this study, it is reported by the company that (a) most of their sales personnel are paid on a commission basis and (b) the firm has to design an effective incentive mechanism for its salesforce and regularly evaluate its effectiveness: "Our objective is to continually evaluate our sales force,..., and utilize appropriate measurements to assess our selling effectiveness" ([13]).

To gain insights into how firms can effectively integrate their production/inventory decisions with the design of effective compensation plans, we develop a dynamic PrincipalAgent model in which the salesforce (representing the agent) sells the product on behalf of the firm (representing the principal). We consider a scenario in which the manager of the firm (she) is risk-neutral and maximizes her expected total profit during the planning horizon, while the sales agent (he) is risk-averse and is myopic in optimizing his short-term utility. In each period, the firm designs a menu of compensation contracts for the agent based on the current level of inventory to (a) induce him to work hard to attract enough demand and (b) extract information about the market condition. The firm also makes production/inventory control decisions based on the extracted information to provide enough supply. The agent, possessing more information about the market than the firm, decides whether to accept an offer, and if so, how much selling effort to exert for that period.

As is the case in most real-world situations, we assume the firm cannot directly monitor the sales effort exerted by the sales agent, and only observes the demand/sales in each period. Thus, the firm has to compensate the agent in each period merely based on sales. Therefore, the problem that the firm is facing in each period is a combination of moral hazard (as the outcome depends on the unobserved agent's effort level) and adverse selection (due to the asymmetric information regarding the market condition). It is well established that an effective approach to this type of problems is to design a menu of contracts ${ }^{1}$ for the agent who, privately knowing the market condition (i.e., the agent type), chooses one to sign (see e.g., [27]). The signed contract, if effective, may induce the agent to work hard enough (to generate enough demand for the firm's stock level). Moreover, by observing the contract signed by the agent, the firm can obtain sufficient information about the market, and can then make use of it for more effective production/inventory decisions (to generate enough supply depending on the market needs).

We first analyze our model in the last period of the planning horizon, and completely characterize the optimal menu of inventory-dependent contracts that should be offered by the firm. We find that the optimal contracts are (1) more attractive when the inventory level is high or the salesman is less riskaverse, (2) effective in inducing the salesforce to work harder than usual when the inventory level is higher, and (3) suitable in providing sufficient information for the firm to effectively make production/inventory decisions. Point (1) establishes analytical support for the widely observed behavior of firms in offering higher commission rates when facing high levels of over-stock inventories. It also supports the empirical studies that find (a) changing compensation plans is effective in increasing sales (see e.g., [31]), and (b) more risk-averse individuals prefer a fixed salary and are less productive under incentive pays (see e.g., [6]; and for more general empirical studies on the effect of incentives, see [42]). Points (2) and (3) shed light on two typically ignored aspects of a good compensation plan.

We then analyze the problem in an arbitrary period. To this end, we focus on the case where the contract does not provide any long-term employment guarantee for the agent, and as a result, the agent (unlike the firm) is mainly concerned with short-term gains. Focusing on these types of contracts, which are prevalently observed in today's competitive business

${ }^{1}$ For a real-world example of the use of menu of contracts, see [1] which provides a report on the menu of incentive contracts commissioned by the Norwegian Water Resources and Energy Directorate. 
where sales agents lack job security, enables us to apply the revelation principle and to completely characterize the optimal dynamic inventory-dependent contracts in each period. We point out that if the contract offers a long-term employment guarantee, or the agent is not myopic, then the agent will become strategic, ${ }^{2}$ and the revelation principle may fail (see e.g., $[28,36,38])$. In that case, our results can serve as an approximation when the agent is relatively more concerned with his short-term gains, but in general, completely characterizing the optimal dynamic inventory-dependent contracts with strategic agent is a difficult task. As we will see, even when the contract does not provide a long-term employment guarantee (e.g., lack of job security in a competitive market), the structure of the inventory-dependent optimal contracts in the multiperiod setting is complex. Nevertheless, we are able to completely characterize the menu and show that the optimal menu of contracts bear a similar (though much more complex) structure compared to the last period.

After characterizing the optimal menu of contracts for an arbitrary period and observing its complexity, we propose a heuristic menu of inventory-dependent contracts using the result for the optimal contracts in the last period. We show the effectiveness of the proposed heuristic through numerical examples. We also illustrate and highlight the importance of using the inventory-level dependent contracts by numerically comparing their performance with those that ignore on-hand inventories. Our analyses enable us to rigorously demonstrate why higher inventory levels result in higher salesforce incentives offered by firms, a phenomenon that has only been empirically observed in practice. ${ }^{3}$

Furthermore, by analyzing the problem in an arbitrary period, we show for the first time in the literature that although the inventory/production control and the design of the compensation package are highly interconnected, firms do not need to implement a complex inventory/production management policy; the optimal inventory control policy is a state-dependent policy that benefits from the extracted market information from the agent (which we refer to as a marketbased state-dependent policy). In addition, for periods with low inventory levels, this policy reduces to a traditional but market-based base-stock policy, in which the firm follows a base-stock policy after making use of the extracted information from the agent. In fact, we find that sales agent screening under optimal contracts enables the firm to implement

\footnotetext{
${ }^{2}$ When the agent is strategic, he might have some incentives to deviate in the first few periods from optimizing his regular utility (the so-called "ratchet effect," see e.g., [20]).

${ }^{3}$ As an example, when gas prices rise, the demand in the auto industry shifts toward more fuel efficient vehicles, and hence, auto-makers face large low-MPG car inventories. As a result, they typically increase their marketing activities for low-MPG cars to speed up their inventory turnover (see e.g., [7]).
}

classic inventory control mechanisms as if the firm has perfect market information (i.e., no information asymmetry).

In closing, we highlight that in contrast to [9] which analyzes a single-period model where the compensation plan offered by the firm is independent of its production/inventory decision, we consider a dynamic setting with features such as nonstationary demand, dynamic market conditions, and inventory carryover, and focus on the important role of inventory decisions in designing a compensation package for the salesforce. Our motivation has been the common empirical observation of firms offering incentives to induce sales agents to work harder than usual during times of inventory overstocking. To the best of our knowledge, our work is the first in the operations literature to provide an analytical justification by rigorously quantifying the significant role of inventory decisions in the design of salesforce compensation.

The rest of the article is organized as follows. We review the literature in Section 2. In Section 3, we present the model, and in Section 4, we study the optimal production and incentive design problem for the last period. In Section 5, we analyze the model for an arbitrary period. Finally in Section 6, we conclude the article with a discussion of our results and highlighting some possible directions for future research. All the proofs are provided in a separate online appendix.

\section{LITERATURE REVIEW}

The authors of [3] are generally considered as the first to apply the agency theory of Economics to study the problem of salesforce compensation and related issues in the Marketing literature. Ref. [3] presents the impact of uncertainty, risk aversion, and some other factors on the design of compensation packages. There are several other studies on the design of salesforce incentives in the Marketing literature. Comprehensive reviews of this stream can be found in [11] and [12]. A typical theme in this stream of research is that the salesforce compensation package should be based on the observed realization of the demand (as a mechanism to measure the salesforce effort). In sharp contrast, the operational decisions such as inventory or production control widely discussed in the OM literature are made a priori and based on some knowledge or prediction about the demand process.

An overview of research on the integration of manufacturing and marketing decisions can be found in [37]. Within this broad stream of research, some studies have tried to fill the gap between the Marketing literature and that of $\mathrm{OM}$ by incorporating the operational decisions such as production/inventory control in the design of incentives. The first work in this area appears to be the interesting study of [2] that considers the incentive design problem between a single owner and a single manager in a newsvendor-type framework. Ref. [41] develops an insightful model to investigate the 
incentive design problem between a manufacturer manager (responsible for making capacity and inventory decisions) and several product managers (responsible for making sales effort decisions).

The author in [8] discusses and evaluates the impact of salesforce incentives (over multiple time periods) on a manufacturing firm's production and inventory decisions, and proposes a moving-window mechanism to induce salespeople to exert considerable selling effort to make the demand process smoother. Ref. [22] studies a setting where a retailer makes ordering and salesforce effort exertion decisions. The authors in [22] find that, similar to the result of [30], the retailer should provide a higher service level to increase the benefit and consequences of learning from uncensored demand information (the so-called "information stalking"). In a single-period salesforce design problem with inventory consideration, [14] also investigates the demand censoring effect and shows that the firm needs to stock slightly more than the usual level, because it helps the firm to gain a better prediction of the agent's hidden effort. The authors of [14] also show that the presence of stockouts may lead the firm to offer higher bonuses to the agent even though the inventory might be limited. Unlike our work, in the analytical setting of [14], demand and the agent's effort only take discrete number of values, and the compensations are quota-based. Ref. [25] also implements the agency theory from Economics to determine compensation plans for sales and operations managers to effectively coordinate their activities. Ref. [15] considers the salesforce compensation design and inventory planning problem when the agent's risk attitude is unknown to the firm.

The author in [9] studies a single-period (i.e., static) problem in which the salesforce compensation problem is separated from the firm's production and inventory decision. Our model builds on and extends [9]. However, our work differs from [9] mainly in two folds: (1) unlike [9], we consider a multiperiod and dynamic setting with inventory carryover, where the market condition, demand, and inventory levels change over time, and (2) we aim to investigate the effect of inventory on the compensation plan, but in [9], the compensation plan is independent of inventory levels. Thus, compared to earlier studies, this work lies in the dynamic nature of the problem and the main interest is in the interplay between inventory and incentive design. To the best of our knowledge, our work is the first attempt in this vein.

Similar to many other studies in the literature, the interaction between the firm and the salesforce in our model is of a Principal-Agent type. These types of models are widely studied and discussed in field of Microeconomics. Refs. [33] and [26] provide excellent descriptions of Principal-Agent type models. Some other books such as [43] and [5] cover broader and more subtle issues regarding the theory of contracts, including those related to strategic commitment and renegotiation in dynamic models.
In general, it is noted that Principal-Agent models in dynamic settings possess several technical challenges, and the underlying theory to handle/investigate those is still immature. This is despite the fact that dynamic PrincipalAgent models can be significantly effective in providing insights into many areas of research, including those in the OM field. One of the first applications of dynamic PrincipalAgent models in OM is [39], which studies a multiperiod setting without adverse selection (i.e., moral hazard only), where the transition probability matrices depend on the actions adopted by the agent. In [39], the principal seeks to make use of a payment mechanism that optimizes her expected discounted profit. Ref. [39] presents a set of assumptions under which two typical difficulties of dynamic Principal-Agent models can be easily handled: (1) history dependence of compensation mechanisms which requires a (non-Markovian) complete information collection over time (i.e., information from all the previous periods) and (2) strategic commitment of both the principal and the agent (i.e., the agreement of both parties at time zero to restrict themselves to the terms for later payments). When (a) the manager's (agent's) utility is an exponential type and additively separable over time, (b) the manager can move consumptions between periods (e.g., through saving and withdrawing income), and (c) the principle is a risk-neutral profit maximizer, [39] shows that the complex problem can be handled using a two-step dynamic programming setting. They also demonstrate that the optimal contract satisfies the Bellman optimality principle/equation.

In another related study, the authors of [40] consider a principal-agent version of the well-known continuous-time make-to-stock queueing system with a single server. They find that an optimal compensation package includes some piece rates as well as dynamic inventory-dependent penalty terms. Refs. [39] and [40] both fall in the class of dynamic moral hazard studies.

Addressing a dynamic Principal-Agent model in an adverse selection context, [46] considers an interesting setting in which the underlying system is a Markov Decision Process, the state of the system can only be monitored/observed by the agent, but the agent's action is not private and can be observed by the principle. In such a setting, [46] establishes that it is sufficient to restrict attention to the revelation contracts. In another study, [45] develops a dynamic adverse selection setting between a supplier and a retailer in a two-echelon supply chain model, where the supplier is unaware of the inventory level at the downstream retailer.

There are also a few studies on dynamic Principal-Agents models with both moral hazard and adverse selection. For this stream of research, we refer interested readers to $[17,18]$, and the references therein. We contribute to this stream of research by considering the salesforce incentive design problem in a setting where the agent does not know the inventory 
of the firm, and the firm cannot observe the effort level exerted by the agent, but she can alter the agent's incentives depending on the inventory level.

In closing this section, we note that while we consider the design of incentive plans for a typical salesperson, recent work on the design of incentives for wholesale-salespersons are also relevant to our study. For this stream of research, we refer interested readers to [24], and the references therein.

\section{THE MODEL}

Consider a firm that sells a single item through a sales agent over $N$ periods (e.g., if $N=4$ it may represent the four quarters of the year). The demand/sales in each period depends on the market condition as well as the effort exerted by the salesforce. The attracted demand in period $n \in\{1,2, \ldots, N\}$ is

$$
D_{n}=\Theta_{n}+a_{n}+\mu_{n}+\epsilon_{n},
$$

where $\Theta_{n}$ is the market condition in period $n, a_{n} \in \mathbb{R}^{+}$is the agent's advertising/sales effort, $\mu_{n}$ is a period-dependent number which may, for example, reflect seasonal effects, and $\epsilon_{n}$ is a random noise which is assumed to be normally distributed with mean 0 and variance $\sigma_{n}^{2}$. The variance in the noise, $\sigma_{n}^{2}$, may depend on period $n$, since in applications the demand may be seasonal and affected by some uncontrolled factors differently in each season. For simplicity, we assume that the effort level exerted by the agent has only a first order affect and does not affect $\sigma_{n}^{2}$.

For tractability and also to be consistent with the literature (see e.g., [9]), we assume that the market can be in either of two possible conditions, High (H) or Low (L), which is equivalent to $\Theta_{n}$ taking two possible values, $\theta_{\mathrm{H}}$ and $\theta_{\mathrm{L}}$ with $\theta_{\mathrm{H}} \geq \theta_{\mathrm{L}}$. At the beginning of each period, the salesman, being closer to the market, learns the market condition, while the firm only has an estimate of the market condition (i.e., some prior belief that the market condition is of type $\mathrm{H}$ or $\mathrm{L}$ ). We assume the market condition process $\left\{\Theta_{n}, n=1,2, \ldots, N\right\}$ evolves as a two-state Markov chain with transition probabilities $\rho_{i j}$ for $i, j=\mathrm{L}, \mathrm{H}$. That is,

$$
\begin{aligned}
& P\left\{\Theta_{n+1}=\theta_{\mathrm{H}} \mid \Theta_{n}=\theta_{\mathrm{H}}\right\}=\rho_{\mathrm{HH}}, \\
& P\left\{\Theta_{n+1}=\theta_{\mathrm{L}} \mid \Theta_{n}=\theta_{\mathrm{H}}\right\}=\rho_{\mathrm{HL}}, \\
& P\left\{\Theta_{n+1}=\theta_{\mathrm{H}} \mid \Theta_{n}=\theta_{\mathrm{L}}\right\}=\rho_{\mathrm{LH}}, \\
& P\left\{\Theta_{n+1}=\theta_{\mathrm{L}} \mid \Theta_{n}=\theta_{\mathrm{L}}\right\}=\rho_{\mathrm{LL}} .
\end{aligned}
$$

The salesman's utility in each period depends on the compensation he receives from the firm as well as the sales effort he exerts. If $s_{i}(D)$ is the compensation for a type $i \in\{\mathrm{H}, \mathrm{L}\}$ agent when the realized sales is $D$, and $a_{i}$ is his exerted effort level, then the salesman's utility during the period is represented by the negative exponential function $u\left(s_{i}(D), a_{i}\right)=-e^{-\gamma\left(s_{i}(D)-v\left(a_{i}\right)\right)}$, where $v\left(a_{i}\right)$ is an increasing $^{4}$ convex function representing the agent's cost of exerting effort/advertising level $a_{i}$, and $\gamma$ is a measure of risk attitude. Similar to some studies in the literature (e.g., [9]), we consider a quadratic form and assume that $v(a)=a^{2} / 2$, which is an example of an increasing convex function; however, our analysis can be extended to other forms of effort cost functions. Negative exponential utility (which is increasing concave) is prevalent in literature, and it is a natural choice of utility when a person's utility is invariant under any wealth translation (i.e., $u(w+k)=f(k) u(w)$ for any wealth $w$ and $k$, and a function $f(\cdot))$. In our framework, the agent maximizes his expected utility in each period and is willing to accept a compensation contract if, and only if, his utility in each period can reach a minimum level $-U_{0}$.

For consistency with the literature, we follow the notation and assumptions of [9], which (unlike our work) studies a single-period problem. In each period, the firm incurs a unit cost $c$ to purchase/produce one unit of the underlying item, and the selling revenue per item is $1+c$, where the marginal profit is assumed to be normalized to one (these parameters can be extended to the case that they are period dependent, see the last section for a discussion). If demand in a period is less than the inventory level, there is a holding cost of $h$ per unit. However, if the demand exceeds the on-hand inventory, then the excess demand must be satisfied via an emergency order at a cost of $p$ per unit. We note that similar assumptions have also been made in several other studies. For instance, in [32], when stockout occurs, it is assumed that the firm borrows inventory from an alternative source. It is noted that this is equivalent to a lost-sales scenario, with the emergency ordering cost being the shortage cost. These cost parameters satisfy $h<c<p<1+c$, but some other cases can also be easily handled, and we allow for some relaxation in our numerical experiments.

The firm is risk-neutral and her objective is to offer a menu of contracts to the salesperson dynamically to maximize her expected total profit (sales revenue minus compensation and production/inventory costs) over a planning horizon of $N$ periods. We note that dynamic/frequent adjustments to incentives (as a tool to increase sales) have been used by firms in practice, see for example, [16] for some related empirical observations in the retail industry and [31] for more general discussions about such adjustments.

As we discussed in the Introduction, the framework for the interaction between the firm and the salesman described above is of a dynamic principal-agent type: the salesman in each period attracts demand for the underlying item on behalf

\footnotetext{
${ }^{4}$ Throughout the article, we use increasing and decreasing in nonstrict sense, that is, they represent nondecreasing and nonincreasing, respectively.
} 
of the firm. In each period, the principal (i.e., the firm) designs a menu of compensation contracts for the agent (i.e., the salesman) and makes production/ordering decisions, while the agent, privately knowing the market condition (which dynamically changes over time), decides whether to accept a specific contract, and if so, how much advertising/selling effort to exert for the period. In this setting, through offering a suitable menu of contracts for each period, the firm would like to (1) invoke the salesman to work hard enough to attract sufficient demand (depending on the inventory level) and (2) extract information about the market condition that can be used for a better production/ordering decision.

The sequence of events in each period is as follows. (1) The firm reviews the inventory level (at the beginning of the period), and based on that and her belief about the market condition, the firm offers a menu of compensation contracts to the agent. (2) The market condition $\theta$ in the period is realized and is privately observed by the agent. (3) The agent decides whether he wants to participate or not, and if he participates, he decides which contract to choose/sign. (4) Under a signed contract, the firm decides the production quantity and the agent chooses the sales/advertising effort level. (5) The firm and the agent both observe the total sales (i.e., the realized value of demand), and the firm receives the selling revenue and pays the compensation to the agent. (6) The inventory shortage or overage cost is accrued for the period, and the system (including the market condition) proceeds to the next period. The resulting problem is a dynamic mechanism design problem. It should be noted that, in general, dynamic mechanism design is very challenging, and the usual revelation principle may fail to hold (see e.g., the discussions in [36]). In this article, we focus on the set of contracts that do not provide any employment guarantee for the sales agent in the future periods. Under such contracts (which represent a competitive market for the agents with lack of job security), the agent is mainly concerned with short-term gains, and the usual revelation principle can be applied. Thus, our approach provides an approximation for analysis of contracts that provide short- to middle-term employment guarantees.

\section{ANALYSIS OF THE LAST PERIOD}

To gain insights, we start by analyzing the problem in the last period, $N$. For simplicity here we suppress the time index denoting the last stage. We also assume that after period $N$ there will be no cost or salvage value. That is, at the end of period $N$, the usual holding and shortage cost incurs and there is no additional cost afterwards. Our notation and analysis in this section is similar to that of [9] (see also [27]) which considers a single-period model, except that we have an arbitrary starting inventory level in the last period, while in [9] the initial inventory level is zero (i.e., ignored). That is, unlike
[9], the optimal contracts in our framework will be inventory dependent. Our goal is to gain insight into the important role of the starting inventory level in contract design. In the next section, we will analyze the problem for an arbitrary period.

Suppose that at the beginning of period $N$, and before any decision is made, the firm's inventory level is $x$, and the salesman privately observes the market condition, $\theta_{\mathrm{H}}$ or $\theta_{\mathrm{L}}$. For convenience, we call the salesman who observes $\theta_{\mathrm{H}}\left(\theta_{\mathrm{L}}\right)$ the $\mathrm{H}$ (L) type agent. By the revelation principle (see e.g., [35]), the firm needs to only consider the class of truth-telling contracts.

Under a signed contract $s_{i}(D)$, to decide how much effort to exert, the type $i \in \mathrm{H}, \mathrm{L}$ agent needs to solve the optimization problem

$$
\max _{a_{i}} \mathbb{E}\left[u\left(s_{i}(D), a_{i}\right)\right]=\max _{a_{i}} \mathbb{E}\left[-e^{-\gamma\left(s_{i}(D)-v\left(a_{i}\right)\right)}\right] .
$$

The compensation scheme we consider is the most commonly used form of compensation, that is, an affine form (see [27] for the optimality of these types of contracts), which represents a commission rate of $\alpha_{i}$ plus a fixed/salary payment of $\beta_{i}: s_{i}(D)=\alpha_{i} D+\beta_{i}$. (As will be seen, the commission rate and the salary depend on the firm's starting inventory level. But here we suppress such a dependency for notational simplicity.) By the certainty equivalence principle, since $s_{i}(D)$ is normally distributed, the optimization problem (1) has the solution

$$
\begin{aligned}
a_{i}^{*}(\theta) & \triangleq \underset{a_{i}}{\operatorname{argmax}} \mathbb{E}\left[-e^{-\gamma\left[s_{i}(D)-a_{i}^{2} / 2\right]}\right] \\
& =\underset{a_{i}}{\operatorname{argmax}}\left\{\mathbb{E}\left[s_{i}(D)\right]-\frac{\gamma}{2} \operatorname{Var}\left[s_{i}(D)\right]-a_{i}^{2} / 2\right\} .
\end{aligned}
$$

The type $i$ agent has the choice of selecting contract $s_{H}$ or $s_{L}$. If he selects contract $j(=\mathrm{L}$ or $\mathrm{H})$, then his optimal effort level is

$$
\begin{aligned}
a^{*}\left(s_{j}, i\right)= & \underset{a_{i}}{\operatorname{argmax}} \mathbb{E}\left[s_{j}(D)\right]-\frac{1}{2} \gamma \operatorname{Var}\left[s_{j}(D)\right]-\frac{a_{i}^{2}}{2} \\
= & \underset{a_{i}}{\operatorname{argmax}} \alpha_{j}\left(\theta_{i}+\mu+a_{i}\right)+\beta_{j}-a_{i}^{2} / 2 \\
& -\frac{1}{2} \gamma \alpha_{j}^{2} \sigma^{2} \\
= & \alpha_{j} .
\end{aligned}
$$

Therefore, if the salesman observes the market condition $i$ and selects contract $s_{j}$, then his optimal effort level to be exerted is $\alpha_{j}$ (see also [9]). The certainty equivalence of the salesperson's expected utility, denoted by $\hat{u}\left(s_{j}, i\right)$, is therefore

$$
\begin{aligned}
\hat{u}\left(s_{j}, i\right) & =\alpha_{j}\left(\theta_{i}+\mu+\alpha_{j}\right)+\beta_{j}-\frac{1}{2} \alpha_{j}^{2}-\frac{1}{2} \gamma \alpha_{j}^{2} \sigma^{2} \\
& =\left(\theta_{i}+\mu\right) \alpha_{j}+\beta_{j}+\frac{1-\gamma \sigma^{2}}{2} \alpha_{j}^{2} .
\end{aligned}
$$


To be able to extract information about the market condition, the firm, when designing a compensation package, needs to consider an incentive compatibility (IC) condition to ensure that the salesman who observes market condition $i$ will indeed select contract $s_{i}$. This entails $\hat{u}\left(s_{i}, i\right) \geq \hat{u}\left(s_{j}, i\right)$ or

$$
\begin{aligned}
& \left(\theta_{i}+\mu\right) \alpha_{i}+\beta_{i}+\frac{1-\gamma \sigma^{2}}{2} \alpha_{i}^{2} \\
& \quad \geq\left(\theta_{i}+\mu\right) \alpha_{j}+\beta_{j}+\frac{1-\gamma \sigma^{2}}{2} \alpha_{j}^{2}, \quad i \neq j .
\end{aligned}
$$

Furthermore, for the salesperson to sign one of the contracts, the firm has to impose an individual rationality (IR) condition to ensure that the selected contract provides a minimum utility value of $-U_{0}$, or a minimum certainty equivalent utility of $-\left(\ln U_{0}\right) / \gamma$, for the agent.

To analyze the firm's problem, we let $\pi_{s}$ be the firm's maximum expected profit under signed contract $s(),$.$x be$ her starting inventory level of the period, and $y \geq x$ be her inventory level right after production (and before demand realization). If $D$ is the demand for the period, then

$$
\begin{aligned}
\pi_{s}= & \max _{y \geq x}\{\mathbb{E}[(1+c) D-s(D)-c(y-x) \\
& \left.\left.-h(y-D)^{+}-p(D-y)^{+}\right]\right\} \\
= & c x+\mathbb{E}[D]-\mathbb{E}[s(D)]-\min _{y \geq x}\left\{(h+c) \mathbb{E}\left[(y-D)^{+}\right]\right. \\
& \left.+(p-c)) \mathbb{E}\left[(D-y)^{+}\right]\right\},
\end{aligned}
$$

where $x^{+}=\max \{x, 0\}$ for any real number $x$. If the demand distribution is known to the firm, and $x=0$, the inventory optimization is a typical newsvendor problem, and its optimal solution, $y^{*}$, is the solution to $P\left\{D \leq y^{*}\right\}=(p-c) /(p+h)$. In particular, if $D$ can be written as $D=v+\epsilon$, where $v$ is a constant (which may depend on parameters such as $\theta, \mu$, and $\alpha_{i}, \beta_{i}$, etc.) and $\in$ is the random noise with cumulative distribution $\Phi$, then the optimal order-up-to level can be written as $y^{*}=v+q^{*}$, where

$$
q^{*}=\Phi^{-1}\left(\frac{p-c}{p+h}\right)
$$

Clearly, $q^{*}$ is the optimal newsvendor solution when the random demand is $\epsilon$, and we shall assume in what follows $q^{*} \geq 0$. If we let $G(y)=(h+c) \mathbb{E}\left[(y-\epsilon)^{+}\right]+(p-$ c) $\mathbb{E}\left[(\epsilon-y)^{+}\right]$, where the expectations are taken with respect to $\in$, then the firm's corresponding optimal cost can be written as $\pi_{s}(x)=c x+\mathbb{E}[D]-\mathbb{E}[s(D)]-G\left((x-v) \vee q^{*}\right)$, where $x \vee y=\max \{x, y\}$ for any real numbers $x$ and $y$.

Suppose in this period the firm offers two contracts $s_{\mathrm{H}}(\cdot)$ and $s_{\mathrm{L}}(\cdot)$ such that the high type agent signs $s_{H}$ and the low type agent signs $s_{L}$. If $s_{H}$ is chosen, then the firm knows that $\Theta=\theta_{\mathrm{H}}$, and therefore, from (3), the agent will exert
$a_{\mathrm{H}}^{*}=\alpha_{\mathrm{H}}$. Hence, it follows from the analysis above that the firm's expected profit, if $s_{H}$ is signed, is

$$
\begin{aligned}
\pi_{s_{\mathrm{H}}}(x)= & c x+\mathbb{E}\left[D \mid \theta=\mathrm{H}, a=a_{\mathrm{H}}\right] \\
& -\mathbb{E}\left[s(D) \mid \theta=\mathrm{H}, a=a_{\mathrm{H}}\right] \\
& \left.-G\left(x-\theta_{\mathrm{H}}-\mu-\alpha_{\mathrm{H}}\right) \vee q^{*}\right) \\
= & c x+\left(1-\alpha_{\mathrm{H}}\right)\left(\theta_{\mathrm{H}}+\mu+\alpha_{\mathrm{H}}\right) \\
& -\beta_{\mathrm{H}}-G\left(\left(x-\theta_{\mathrm{H}}-\mu-\alpha_{\mathrm{H}}\right) \vee q^{*}\right) .
\end{aligned}
$$

Similarly, if contract $s_{L}$ is signed by the agent, the firm's expected profit is

$$
\begin{aligned}
\pi_{s_{\mathrm{L}}}(x)= & c x+\left(1-\alpha_{\mathrm{L}}\right)\left(\theta_{\mathrm{L}}+\mu+\alpha_{\mathrm{L}}\right)-\beta_{\mathrm{L}} \\
& -G\left(\left(x-\theta_{\mathrm{L}}-\mu-\alpha_{\mathrm{L}}\right) \vee q^{*}\right) .
\end{aligned}
$$

The firm offers contracts $s_{H}$ and $s_{L}$. Under the signed contract $s_{i}$, the salesperson's optimal certainty equivalence is

$$
\alpha_{i}\left(\theta_{i}+\mu\right)+\alpha_{i}^{2}\left(1-\gamma \sigma^{2}\right) / 2+\beta_{i} .
$$

Therefore, if the firm believes at the beginning of the period that the market condition will be of type $\mathrm{H}$ with probability $\rho$, then the firm's problem of designing the optimal contract can be written as

$$
\begin{aligned}
& \text { s.t. } \\
& \alpha_{\mathrm{H}}\left(\theta_{\mathrm{H}}+\mu\right)+\alpha_{\mathrm{H}}^{2}\left(1-\gamma \sigma^{2}\right) / 2+\beta_{\mathrm{H}} \\
& \geq\left(-\ln U_{0}\right) / \gamma \quad(\mathrm{IR}-\mathrm{H}) \\
& \alpha_{\mathrm{L}}\left(\theta_{\mathrm{L}}+\mu\right)+\alpha_{\mathrm{L}}^{2}\left(1-\gamma \sigma^{2}\right) / 2+\beta_{\mathrm{L}} \\
& \geq\left(-\ln U_{0}\right) / \gamma \quad(\mathrm{IR}-\mathrm{L}) \\
& \alpha_{\mathrm{H}}\left(\theta_{\mathrm{H}}+\mu\right)+\alpha_{\mathrm{H}}^{2}\left(1-\gamma \sigma^{2}\right) / 2+\beta_{\mathrm{H}} \\
& \geq \alpha_{\mathrm{L}}\left(\theta_{\mathrm{H}}+\mu\right)+\alpha_{\mathrm{L}}^{2}\left(1-\gamma \sigma^{2}\right) / 2 \\
& +\beta_{\mathrm{L}} \quad(\mathrm{IC}-\mathrm{HL}) \\
& \alpha_{\mathrm{L}}\left(\theta_{\mathrm{L}}+\mu\right)+\alpha_{\mathrm{L}}^{2}\left(1-\gamma \sigma^{2}\right) / 2+\beta_{\mathrm{L}} \\
& \geq \alpha_{\mathrm{H}}\left(\theta_{\mathrm{L}}+\mu\right)+\alpha_{\mathrm{H}}^{2}\left(1-\gamma \sigma^{2}\right) / 2 \\
& +\beta_{\mathrm{H}} \quad(\mathrm{IC}-\mathrm{LH}) \\
& \alpha_{\mathrm{H}}, \alpha_{\mathrm{L}} \geq 0 .
\end{aligned}
$$$$
V_{N}(x, \rho)=\max _{\alpha_{\mathrm{H}}, \beta_{\mathrm{H}}, \alpha_{\mathrm{L}}, \beta_{\mathrm{L}}}\left\{c x+\rho\left[\left(\theta_{\mathrm{H}}+\mu+\alpha_{\mathrm{H}}\right)\left(1-\alpha_{\mathrm{H}}\right)\right.\right.
$$$$
\left.-\beta_{\mathrm{H}}-G\left(q^{*} \vee\left[x-\theta_{\mathrm{H}}-\mu-\alpha_{\mathrm{H}}\right]\right)\right]
$$$$
+(1-\rho)\left[\left(\theta_{\mathrm{L}}+\mu+\alpha_{\mathrm{L}}\right)\left(1-\alpha_{\mathrm{L}}\right)-\beta_{\mathrm{L}}\right.
$$

Using the convention in the standard mechanism design literature for labeling the constraints, (8) and (9) are the socalled individual rationality (IR) constraints for the $\mathrm{H}$ type and L type agents, respectively, which guarantee that the agent will participate regardless of his type. Constraints (10) 
and (11) are the so-called incentive compatibility constraints, which guarantee that an $\mathrm{H}(\mathrm{L})$ type agent will not choose/sign a L $(\mathrm{H})$ type contract. Finally, constraint (12) assures that the commission rates offered by the firm (i.e., principal) are nonnegative.

Following the same lines of formulation as [9] (but with the exception that our optimization program depends on the starting inventory level, $x$ ), the above optimization problem can be significantly simplified. To see this, first note that, since $\theta_{\mathrm{H}}>\theta_{\mathrm{L}},(\mathrm{IC}-\mathrm{HL})$ and (IR-L) together result in (IR-H), and hence (IR-H) can be eliminated without any loss of generality. Next, note that (IC-HL) would need to hold as equality (otherwise, decreasing $\beta_{\mathrm{H}}$ will provide a better solution without violating the remaining constraints). Writing (IC-HL) as an equality, observe that (IC-LH) can be substituted with $\alpha_{\mathrm{H}} \geq \alpha_{\mathrm{L}}$, because $\theta_{\mathrm{H}}>\theta_{\mathrm{L}}$. Moreover, note that (IR-L) must hold as an equality because otherwise it is possible to decrease $\beta_{\mathrm{H}}$ and $\beta_{\mathrm{L}}$ by the same amount to obtain a better solution while satisfying all the remaining constraints. Performing the above changes, we obtain the following simpler problem:

$$
\begin{aligned}
V_{N}(x, \rho)= & \max _{\alpha_{\mathrm{H}}, \beta_{\mathrm{H}}, \alpha_{\mathrm{L}}, \beta_{\mathrm{L}}}\left\{c x+\rho\left[\left(\theta_{\mathrm{H}}+\mu+\alpha_{\mathrm{H}}\right)\left(1-\alpha_{\mathrm{H}}\right)\right.\right. \\
& \left.-\beta_{\mathrm{H}}-G\left(q^{*} \vee\left[x-\theta_{\mathrm{H}}-\mu-\alpha_{\mathrm{H}}\right]\right)\right] \\
& +(1-\rho)\left[\left(\theta_{\mathrm{L}}+\mu+\alpha_{\mathrm{L}}\right)\left(1-\alpha_{\mathrm{L}}\right)-\beta_{\mathrm{L}}\right. \\
& \left.\left.-G\left(q^{*} \vee\left[x-\theta_{\mathrm{L}}-\mu-\alpha_{\mathrm{L}}\right]\right)\right]\right\}
\end{aligned}
$$

$$
\begin{array}{ll}
\text { s.t. } & \alpha_{\mathrm{L}}\left(\theta_{\mathrm{L}}+\mu\right)+\alpha_{\mathrm{L}}^{2}\left(1-\gamma \sigma^{2}\right) / 2+\beta_{\mathrm{L}}=\left(-\ln U_{0}\right) / \gamma \\
& \alpha_{\mathrm{H}}\left(\theta_{\mathrm{H}}+\mu\right)+\alpha_{\mathrm{H}}^{2}\left(1-\gamma \sigma^{2}\right) / 2+\beta_{\mathrm{H}} \\
& =\alpha_{\mathrm{L}}\left(\theta_{\mathrm{H}}+\mu\right)+\alpha_{\mathrm{L}}^{2}\left(1-\gamma \sigma^{2}\right) / 2+\beta_{\mathrm{L}} \\
& \alpha_{\mathrm{H}} \geq \alpha_{\mathrm{L}} \geq 0 .
\end{array}
$$

The analysis above shows that, as is typically expected, an optimal menu of contracts makes (1) the high type agent indifferent between the two contracts offered and (2) the low type agent indifferent between signing a contract and not participating (i.e., not signing any contract). We can now use the remaining constraints [i.e., (IC-HL) and (IR-L)] to find the fixed/salary payments $\beta_{\mathrm{H}}$ and $\beta_{\mathrm{L}}$ :

$$
\begin{aligned}
\beta_{\mathrm{L}}= & -\alpha_{\mathrm{L}}\left(\theta_{\mathrm{L}}+\mu\right)-\alpha_{\mathrm{L}}^{2}\left(1-\gamma \sigma^{2}\right) / 2-\left(\ln U_{0}\right) / \gamma \\
\beta_{\mathrm{H}}= & +\alpha_{\mathrm{L}}\left(\theta_{\mathrm{H}}-\theta_{\mathrm{L}}\right)-\alpha_{\mathrm{H}}\left(\theta_{\mathrm{H}}+\mu\right) \\
& -\alpha_{\mathrm{H}}^{2}\left(1-\gamma \sigma^{2}\right) / 2-\left(\ln U_{0}\right) / \gamma .
\end{aligned}
$$

Using these equations and simplifying the objective function, the firm's problem can be rewritten as

$$
\begin{aligned}
& V_{N}(x, \rho)=\max _{\alpha_{\mathrm{H}}, \alpha_{\mathrm{L}}}\left\{c x+\frac{\ln U_{0}}{\gamma}+\bar{\theta}+\mu\right. \\
& +\rho\left[\alpha_{\mathrm{H}}-\frac{1+\gamma \sigma^{2}}{2} \alpha_{\mathrm{H}}^{2}-G\left(q^{*} \vee\left[x-\theta_{\mathrm{H}}-\mu-\alpha_{\mathrm{H}}\right]\right)\right]
\end{aligned}
$$

$$
\begin{aligned}
& +(1-\rho)\left[\alpha_{\mathrm{L}}-\frac{\rho}{1-\rho} \alpha_{\mathrm{L}}\left(\theta_{\mathrm{H}}-\theta_{\mathrm{L}}\right)-\frac{1+\gamma \sigma^{2}}{2} \alpha_{\mathrm{L}}^{2}\right. \\
& \left.\left.-G\left(q^{*} \vee\left[x-\theta_{\mathrm{L}}-\mu-\alpha_{\mathrm{L}}\right]\right)\right]\right\}
\end{aligned}
$$

s.t. $\alpha_{\mathrm{H}} \geq \alpha_{\mathrm{L}} \geq 0$,

where $\bar{\theta}=\rho \theta_{\mathrm{H}}+(1-\rho) \theta_{\mathrm{L}}$.

We note that the objective function of the above optimization program is separable in decision variables $\alpha_{\mathrm{H}}$ and $\alpha_{\mathrm{L}}$ (although these decision variables are connected through the starting inventory level). Moreover, it is easy to check that the function $G(\cdot)$ is convex, and since $q^{*}$ is its minimizer, it follows that $G\left(q^{*} \vee\left[x-\theta_{\mathrm{H}}-\mu-\alpha_{\mathrm{H}}\right]\right)$ and $G\left(q^{*} \vee\left[x-\theta_{\mathrm{L}}-\mu-\alpha_{\mathrm{L}}\right]\right)$ are both convex in $x$. Hence, the above objective function is concave and separable in decision variables.

To solve the mathematical program (15), we first solve two separate optimization problems (i.e., after relaxing the constraint $\alpha_{\mathrm{H}} \geq \alpha_{\mathrm{L}} \geq 0$ ). We first optimize

$$
\alpha_{\mathrm{H}}-\frac{1+\gamma \sigma^{2}}{2} \alpha_{\mathrm{H}}^{2}-G\left(q^{*} \vee\left[x-\theta_{\mathrm{H}}-\mu-\alpha_{\mathrm{H}}\right]\right) .
$$

It can be seen that when $x \leq q^{*}+\theta_{\mathrm{H}}+\mu+1 /\left(1+\gamma \sigma^{2}\right)$, the optimal $\alpha_{\mathrm{H}}$ is equal to $1 /\left(1+\gamma \sigma^{2}\right)$, and when $x \geq$ $q^{*}+\theta_{\mathrm{H}}+\mu+1 /\left(1+\gamma \sigma^{2}\right)$, the optimal $\alpha_{\mathrm{H}}$ is the maximizer of

$$
\alpha_{\mathrm{H}}-\frac{1+\gamma \sigma^{2}}{2} \alpha_{\mathrm{H}}^{2}-G\left(x-\theta_{\mathrm{H}}-\mu-\alpha_{\mathrm{H}}\right),
$$

which is always greater than $1 /\left(1+\gamma \sigma^{2}\right)$. We let $\alpha_{\mathrm{H}}(x)$ be the maximizer of (17).

We then optimize

$$
\begin{aligned}
& \alpha_{\mathrm{L}}-\frac{\rho}{1-\rho} \alpha_{\mathrm{L}}\left(\theta_{\mathrm{H}}-\theta_{\mathrm{L}}\right)-\frac{1+\gamma \sigma^{2}}{2} \alpha_{\mathrm{L}}^{2} \\
&-G\left(q^{*} \vee\left[x-\theta_{\mathrm{L}}-\mu-\alpha_{\mathrm{L}}\right]\right) .
\end{aligned}
$$

When $x \leq q^{*}+\theta_{\mathrm{L}}+\mu+\frac{1-\frac{\rho}{1-\rho}\left(\theta_{\mathrm{H}}-\theta_{\mathrm{L}}\right)}{1+\gamma \sigma^{2}}$, the optimal $\alpha_{\mathrm{L}}$ is equal to $\frac{1-\frac{\rho}{1-\rho} \alpha_{\mathrm{L}}\left(\theta_{\mathrm{H}}-\theta_{\mathrm{L}}\right)}{1+\gamma \sigma^{2}}$, and when $x \geq q^{*}+\theta_{\mathrm{L}}+\mu+$ $\frac{1-\frac{\rho}{1-\rho}\left(\theta_{\mathrm{H}}-\theta_{\mathrm{L}}\right)}{1+\gamma \sigma^{2}}$, the optimal $\alpha_{\mathrm{L}}$ is the maximizer of

$$
\begin{aligned}
\alpha_{\mathrm{L}} & -\frac{\rho}{1-\rho} \alpha_{\mathrm{L}}\left(\theta_{\mathrm{H}}-\theta_{\mathrm{L}}\right)-\frac{1+\gamma \sigma^{2}}{2} \alpha_{\mathrm{L}}^{2} \\
& -G\left(x-\theta_{\mathrm{L}}-\mu-\alpha_{\mathrm{L}}\right),
\end{aligned}
$$

which is always greater than $\frac{1-\frac{\rho}{1-\rho} \alpha_{\mathrm{L}}\left(\theta_{\mathrm{H}}-\theta_{\mathrm{L}}\right)}{1+\gamma \sigma^{2}}$. We let $\alpha_{\mathrm{L}}(x)$ be the maximizer of (18).

From the analysis above, we observe that the optimal menu of contracts may take the same value for both agent types (in 
some range of the starting inventory level). To achieve the benefits of information acquisition, the firm can slightly perturb the contracts so that they are differentiated. For instance, when $\alpha^{*}(x)$ is the optimal commission for both type at the inventory level $x$, the firm can set $\alpha_{\mathrm{H}}^{*}(x)=\alpha^{*}(x)+v$ and $\alpha_{\mathrm{L}}^{*}(x)=\alpha^{*}(x)-v$ for some arbitrarily small $v>0$. We will denote these quantities by $\alpha^{*}(x)_{+}$and $\alpha^{*}(x)_{-}$, respectively.

The following properties of $\alpha_{\mathrm{H}}(x)$ and $\alpha_{\mathrm{L}}(x)$ are useful in characterizing the optimal menu of contracts and understanding the form of optimal inventory-dependent commissions that should be offered by the firm.

LEMMA 1 (High Over-Stock Inventories): On $x \geq q^{*}+$ $\theta_{\mathrm{H}}+\mu+1 /\left(1+\gamma \sigma^{2}\right)$, we have

a. $\alpha_{\mathrm{H}}(x)$ and $\alpha_{\mathrm{L}}(x)$ are strictly increasing in $x$ but with slopes less than 1;

b. $\alpha_{\mathrm{H}}(x)$ and $\alpha_{\mathrm{L}}(x)$ both converge to finite values as $x \rightarrow \infty$, and

$$
\begin{aligned}
\lim _{x \rightarrow \infty} \alpha_{\mathrm{L}}(x)<\lim _{x \rightarrow \infty} \alpha_{\mathrm{H}}(x) \\
=\lim _{x \rightarrow \infty} \alpha_{\mathrm{L}}(x)+\frac{\frac{\rho}{1-\rho}\left(\theta_{\mathrm{H}}-\theta_{\mathrm{L}}\right)}{1+\gamma \sigma^{2}} ;
\end{aligned}
$$

c. $\alpha_{\mathrm{H}}(x)$ and $\alpha_{\mathrm{L}}(x)$ are concave in $x$;

d. $\alpha_{\mathrm{H}}(x)-\alpha_{\mathrm{L}}(x)$ is strictly increasing in $x$.

The results above partially reveal the effect of the starting inventory level: when the firm is facing high over-stock inventories, commissions offered are increasing in the inventory level, the $\mathrm{H}$ type agent gets a higher commission rate than the L type agent, and the difference between commission rates becomes larger as the inventory level increases. The latter result suggests that a firm with higher levels of over-stock inventory needs to be more careful in differentiating between agent types: the firm needs to ensure that the $\mathrm{H}$ type agent has enough incentives to work hard to bring the inventory level down while knowing that the L type agent has only a limited leverage in doing so (due to low demands).

To provide a complete characterization of the optimal contracts, we need to provide some preliminary results. First, we need to define the following belief threshold:

$$
\rho^{*}=\frac{\phi\left(q^{*}\right)}{1+\phi\left(q^{*}\right)},
$$

where $\phi$ is the density function of the Normal random variable $\epsilon$ (and hence, $0 \leq \rho^{*} \leq 1$ ). We then define another function by

$$
\xi(\rho, \theta)=\Phi^{-1}\left(\frac{p-c+\frac{\rho}{1-\rho} \theta}{p+h}\right)-\Phi^{-1}\left(\frac{p-c}{p+h}\right),
$$

and provide the following lemma.

Naval Research Logistics DOI 10.1002/nav
LEMMA 2: If $\rho<\rho^{*}$, then there exists a unique number $\hat{\theta}(\rho)$ satisfying $0<\hat{\theta}(\rho)<\infty$, such that $\theta>\xi(\rho, \theta)$ if, and only if, $\theta \leq \hat{\theta}(\rho)$. If $\rho \geq \rho^{*}$, then $\theta \leq \xi(\rho, \theta)$ for all $\theta \geq 0$.

We now present two definitions that will facilitate our characterization of the optimal compensation plans.

DEFINITION 1 (Perceived Market Conditions): We say that the market condition is perceived to be of high (low) type by the firm if $\rho \geq(<) \rho^{*}$ [where $\rho^{*}$ is the belief threshold defined in (19)].

DEFINITION 2 (Distinguishable Market Conditions): The market conditions are said to be distinguishable by the firm if, and only if, $\theta_{\mathrm{H}}-\theta_{\mathrm{L}}>\tilde{\theta}(\rho)$, where $\tilde{\theta}(\rho)$ is the unique solution to $(p+h) \Phi\left(\tilde{\theta}+q^{*}\right)=p-c-\tilde{\theta} \rho /(1-\rho)$.

Using the above definitions, the following result sheds light on the conditions under which relaxing the constraint of program (15) and separably optimizing the objective function for finding the optimal commission rates for $\mathrm{L}$ and $\mathrm{H}$ type agents is or is not problematic.

LEMMA 3 (Single Crossing): If either (a) the market condition is perceived to be of high type or (b) the market condition is perceived to be of low type but market conditions are distinguishable, then there exists a finite number $z^{*}>q^{*}+\theta_{\mathrm{H}}+\mu+1 /\left(1+\gamma \sigma^{2}\right)$ such that $\alpha_{\mathrm{H}}(x)<\alpha_{\mathrm{L}}(x)$ on $z^{*}>x \geq q^{*}+\theta_{\mathrm{H}}+\mu+1 /\left(1+\gamma \sigma^{2}\right), \alpha_{\mathrm{H}}\left(z^{*}\right)=\alpha_{\mathrm{L}}\left(z^{*}\right)$, and $\alpha_{\mathrm{H}}(x)>\alpha_{\mathrm{L}}(x)$ on $x>z^{*}$. Otherwise, $\alpha_{\mathrm{H}}(x) \geq \alpha_{\mathrm{L}}(x)$ on all $x \geq q^{*}+\theta_{\mathrm{H}}+\mu+1 /\left(1+\gamma \sigma^{2}\right)$.

Before completely characterizing the optimal inventorydependent menu of contracts, we also need the following simple result. Its proof is elementary and is omitted.

LEMMA 4: Let $g_{1}(x)$ and $g_{2}(y)$ be two concave functions with maximizers $x^{*}$ and $y^{*}$, respectively. Let $z^{*}$ be the maximizer of concave function $g_{1}(z)+g_{2}(z)$. For the program $\max _{x \geq y}\left\{g_{1}(x)+g_{2}(y)\right\}$ the following result hold: If $x^{*} \geq y^{*}$, then the optimal solution is $\left(x^{*}, y^{*}\right)$; and if $x^{*}<y^{*}$, the optimal solution is $\left(z^{*}, z^{*}\right)$, where $x^{*}<z^{*}<y^{*}$.

We are now ready to characterize the optimal inventorydependent contracts to be offered by the firm. For the ease of notation, we let $\delta=\left[1-\frac{\rho}{1-\rho}\left(\theta_{\mathrm{H}}-\theta_{\mathrm{L}}\right)\right]^{+}$, and define the inventory level intervals

$$
\begin{aligned}
& I_{1}=\left(-\infty, q^{*}+\theta_{\mathrm{L}}+\mu+\delta\left(1+\gamma \sigma^{2}\right)^{-1}\right), \\
& I_{2}=\left[q^{*}+\theta_{\mathrm{L}}+\mu+\delta\left(1+\gamma \sigma^{2}\right)^{-1}, z_{1}^{*}\right), \\
& I_{3}=\left[z_{1}^{*}, z_{2}^{*}\right), I_{4}=\left[z_{2}^{*}, z_{3}^{*}\right), I_{5}=\left[z_{3}^{*}, \infty\right), \\
& I_{1}^{\prime}=\left(-\infty, q^{*}+\theta_{\mathrm{L}}+\mu+\delta\left(1+\gamma \sigma^{2}\right)^{-1}\right),
\end{aligned}
$$




$$
\begin{aligned}
I_{2}^{\prime}= & {\left[q^{*}+\theta_{\mathrm{L}}+\mu+\delta\left(1+\gamma \sigma^{2}\right)^{-1},\right.} \\
& \left.q^{*}+\theta_{\mathrm{H}}+\mu+\left(1+\gamma \sigma^{2}\right)^{-1}\right), \\
I_{3}^{\prime}= & {\left[q^{*}+\theta_{\mathrm{H}}+\mu+\left(1+\gamma \sigma^{2}\right)^{-1}, \infty\right), }
\end{aligned}
$$

where

$$
\begin{aligned}
z_{1}^{*}= & \theta_{\mathrm{L}}+\mu+\left(1+\gamma \sigma^{2}\right)^{-1} \\
& +\Phi^{-1}\left(\frac{p-c+\frac{\rho}{1-\rho}\left(\theta_{\mathrm{H}}-\theta_{\mathrm{L}}\right)}{p+h}\right)
\end{aligned}
$$

$z_{2}^{*}$ is the solution to $z_{2}-\theta_{\mathrm{H}}-\mu-\tilde{\alpha}_{\mathrm{H}}\left(z_{2}\right)=q^{*}$, and $z_{3}^{*}$ is the solution to $\tilde{\alpha}_{\mathrm{H}}\left(z_{3}\right)=\tilde{\alpha}_{\mathrm{L}}\left(z_{3}\right)$, where $\tilde{\alpha}_{\mathrm{H}}(x)$ is the solution to

$$
\begin{aligned}
& (p+h) \Phi\left(x-\theta_{\mathrm{H}}-\mu-\tilde{\alpha}_{\mathrm{H}}\right)-\left(1+\gamma \sigma^{2}\right) \tilde{\alpha}_{\mathrm{H}} \\
& \quad-p+c+1=0
\end{aligned}
$$

and $\tilde{\alpha}_{\mathrm{L}}(x)$ is the solution to

$$
\begin{gathered}
(p+h) \Phi\left(x-\theta_{\mathrm{L}}-\mu-\tilde{\alpha}_{\mathrm{L}}\right)-\left(1+\gamma \sigma^{2}\right) \tilde{\alpha}_{\mathrm{L}} \\
-p+c+1-\frac{\rho}{1-\rho}\left(\theta_{\mathrm{H}}-\theta_{\mathrm{L}}\right)=0 .
\end{gathered}
$$

With these, we can now characterize the optimal menu of inventory-dependent contracts as follows.

THEOREM 1 (Optimal Commissions-Last Period): (1) If either (a) the market condition is perceived to be of high type or (b) the market condition is perceived to be of low type but the market conditions are distinguishable by the firm, then the optimal inventory-dependent commissions are as follows:

1. If $x \in I_{1}$, then $\alpha_{\mathrm{L}}^{*}(x)=\delta /\left(1+\gamma \sigma^{2}\right)$ and $\alpha_{\mathrm{H}}^{*}(x)=$ $1 /\left(1+\gamma \sigma^{2}\right)$

2. if $x \in I_{2}$, then $\alpha_{\mathrm{L}}^{*}(x)=\tilde{\alpha}_{\mathrm{L}}(x)$ and $\alpha_{\mathrm{H}}^{*}(x)=$ $\left(1+\gamma \sigma^{2}\right)^{-1}$;

3. if $x \in I_{3}$, then $\alpha_{\mathrm{L}}^{*}(x)=\alpha_{I_{3}}^{*}(x)_{-}$and $\alpha_{\mathrm{H}}^{*}(x)=$ $\alpha_{I_{3}}^{*}(x)_{+}$, where $\alpha_{I_{3}}^{*}(x)$ is the solution to $(p+h)(1-$ $\rho) \Phi\left(x-\theta_{\mathrm{L}}-\mu-\alpha_{I_{3}}\right)-\left(1+\gamma \sigma^{2}\right) \alpha_{I_{3}}-(1-\rho)(p-$ c) $-\rho\left(\theta_{\mathrm{H}}-\theta_{\mathrm{L}}\right)+1=0$;

4. if $x \in I_{4}$, then $\alpha_{\mathrm{L}}^{*}(x)=\alpha_{I_{4}}^{*}(x)_{-}$and $\alpha_{\mathrm{H}}^{*}(x)=$ $\alpha_{I_{4}}^{*}(x)_{+}$, where $\alpha_{I_{4}}^{*}(x)$ is the solution to $(p+$ $h)\left[\rho \Phi\left(x-\theta_{\mathrm{H}}-\mu-\alpha_{I_{4}}\right)+(1-\rho) \Phi\left(x-\theta_{\mathrm{L}}-\mu-\right.\right.$ $\left.\left.\alpha_{I_{4}}\right)\right]-\left(1+\gamma \sigma^{2}\right) \alpha-p+c-\rho\left(\theta_{\mathrm{H}}-\theta_{\mathrm{L}}\right)+1=0$;

5. if $x \in I_{5}$, then $\alpha_{\mathrm{L}}^{*}(x)=\tilde{\alpha}_{\mathrm{L}}(x)$ and $\alpha_{\mathrm{H}}^{*}(x)=\tilde{\alpha}_{\mathrm{H}}(x)$.

(2) Otherwise, the optimal commissions are as follows:

1. If $x \in I_{1}^{\prime}$, then $\alpha_{\mathrm{L}}^{*}(x)=\delta\left(1+\gamma \sigma^{2}\right)^{-1}$ and $\alpha_{\mathrm{H}}^{*}(x)=$ $\left(1+\gamma \sigma^{2}\right)^{-1}$;
2. if $x \in I_{2}^{\prime}$, then $\alpha_{\mathrm{L}}^{*}(x)=\tilde{\alpha}_{\mathrm{L}}(x)$ and $\alpha_{\mathrm{H}}^{*}(x)=$ $\left(1+\gamma \sigma^{2}\right)^{-1}$;

3. if $x \in I_{3}^{\prime}$, then $\alpha_{\mathrm{L}}^{*}(x)=\tilde{\alpha}_{\mathrm{L}}(x)$ and $\alpha_{H}^{*}(x)=\tilde{\alpha}_{H}(x)$.

The optimal commissions presented above are complex. But we can gain insights by developing a numerical study and illustrating the dependency of the optimal commissions to the firm's inventory level.

Numerical Study 1. Figure 1 illustrates the optimal inventory-based commissions offered by a firm to its salesman as well as the associated utilities. The system parameters are $p=7, h=1, c=2, \sigma=1, \mu=0, U_{0}=10, \gamma=2$, $\theta_{\mathrm{H}}=5$, and $\theta_{\mathrm{L}}=1$. In this figure, the column on left is for a case where the firm's belief about the market (or the agent) being of type $\mathrm{H}$ is $\rho=0.9$, and the column on right illustrates a case where $\rho=0.3$. In the latter case, the structure of the optimal commissions depends on five inventory intervals, as predicted by Theorem 1 . In the former case, as can be seen in Fig. 1a, the firm, regardless of her inventory level, only offers a fixed/salary payment to the low type agent (i.e., $\alpha_{\mathrm{L}}^{*}(x)=0$ for all $x \geq 0$ ). However, in the case with $\rho=0.3$, the $\mathrm{L}$ type agent is offered a salary-only payment only when the inventory level is low (Fig. 1b). Nevertheless, as Figs. 1c and $1 \mathrm{~d}$ show, in both cases with $\rho=0.3$ and $\rho=0.9$ the L type agent's utility is always equal to the minimum level that will make him participate, regardless of the inventory level.

The following result presents conditions under which the low type agent is only offered a fixed/salary payment.

COROLLARY 1 (Salary-Only Payment): If $\rho \geq(h+c+$ 1) $/\left(h+c+1+\left(\theta_{\mathrm{H}}-\theta_{\mathrm{L}}\right)\right)$ then $\alpha_{\mathrm{L}}^{*}(x)=0$ for all $x$, that is, the low type agent is only offered a salary payment regardless of the firm's inventory level. Furthermore, when $x \leq q^{*}+\theta_{\mathrm{L}}+\mu+\delta\left(1+\gamma \sigma^{2}\right)^{-1}$, the low type agent is only offered a salary payment if, and only if, $\rho \geq 1 /\left(1+\left(\theta_{\mathrm{H}}-\theta_{\mathrm{L}}\right)\right)$.

The above result shows that when the firm is sure enough that the market is high, she does not offer a commissionbased payment to the low type agent. In that case, from (13), the firm only offers the minimum salary that would make the low type agent indifferent between signing the contract and leaving.

The following result sheds more light on the dependency of incentives on inventory and risk attitude.

COROLLARY 2: (Monotone and Locally Concave Commissions) The optimal commissions to be offered by the firm are (1) increasing in the firm's inventory level, (2) decreasing in the agent's risk aversion level, and (3) concave in each inventory range.

Parts (1) and (2) of the above results illuminate the interplay between the inventory level and optimal contracts. 


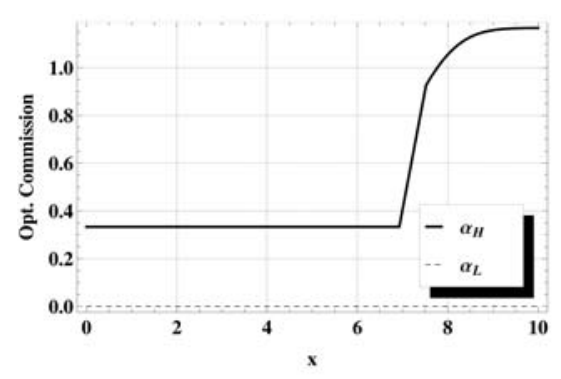

(a) Optimal Commissions $(\rho=0.9)$

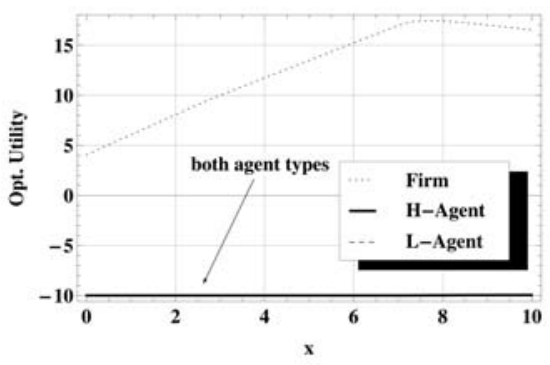

(c) Optimal Utilities $(\rho=0.9)$

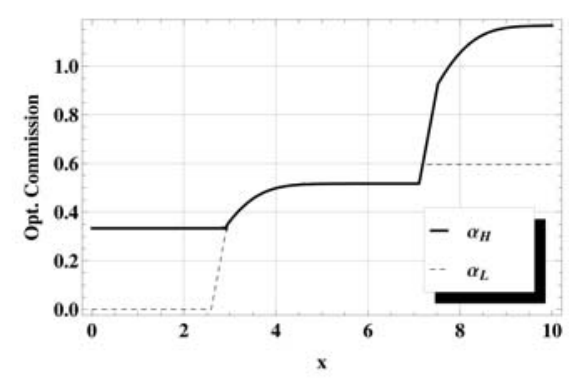

(b) Optimal Commissions $(\rho=0.3)$

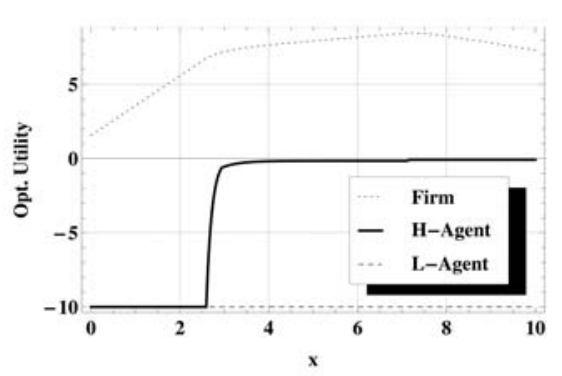

(d) Optimal Utilities $(\rho=0: 3)$

Figure 1. Optimal commissions and the associated utilities.

Specifically, they show that (a) optimal contracts are indeed more attractive $\mathrm{e}^{5}$ when inventory levels are high, and (b) the firm needs to offer better commissions to its less risk-averse agents. These findings strongly support the empirical studies reported in the literature that find (i) varying compensation plans is effective in increasing sales (see e.g., [31]) and (ii) more risk-averse individuals prefer a fixed salary and are less productive under incentive pays (see e.g., [6] and for more general empirical studies on the effect of incentives see [42]). Part 3 of Corollary 2 states that the commission rates possess a local diminishing rate of increase property; for each inventory interval, the rate of increase in the agent's commission is decreasing in the firm's inventory level.

Another interesting insight from the optimal menu of inventory-dependent contracts is that it successfully induces the agent to exert more effort when the inventory is higher. In fact, as Corollary 2 indicates, with a higher inventory level, the firm offers a higher commission rate. And the next result states that, this will induce the agent to work harder to attract more demand. Hence, inventory-dependent contract design is an effective tool to match demand with supply.

COROLLARY 3 (Monotone Effort Level): The agent's optimal effort level induced by the firm is increasing in the firm's inventory level.

\footnotetext{
${ }^{5}$ For convenience, we call a contract more attractive (to the agent) if the commission rate offered (by the firm) is higher.
}

Another simple but important result that we will use in the next section is the following.

PROPOSITION 1: (Concave Value Function-Last Period) The firm's optimal expected profit as a function of the starting inventory $x$ and belief probability $\rho$, denoted by $V_{N}(x, \rho)$ and defined in (15), is concave in $x$ for any fixed $\rho$. Furthermore, $V_{N}(x, \rho)-c x$ is decreasing in $x$ for any fixed $\rho$.

This result shows that, similar to what classic inventory models predict, inventory has a diminishing rate of return effect on the firm's profit: the rate of increase in the firm's profit is (a) decreasing in its inventory and (b) bounded above by the purchasing/production cost, $c$.

\section{ANALYSIS OF AN ARBITRARY PERIOD}

We now proceed to study the joint contract design and inventory/production control problem for an arbitrary period $n, n<N$.

By backward induction, suppose that we have analyzed the problem from stage $n+1$ to the last stage. In particular, suppose we have computed the firm's maximum expected profit from period $n+1$ to the end of the planning horizon, $V_{n+1}\left(x_{n+1}, \rho_{n+1}\right)$, which depends on the state of the system $\left(x_{n+1}, \rho_{n+1}\right)$ at the beginning of period $n+1$, where $x_{n+1}$ is the starting inventory level of period $n+1$ and $\rho_{n+1}$ is the firm's belief probability that the market condition in period $n+1$ will be high $(\mathrm{H})$. We now solve the firm's problem at 
the beginning of period $n$. Recall that, in period $n$, the salesman first learns of the market condition, but the firm only has a belief probability that it is high $(\mathrm{H})$ or low $(\mathrm{L})$. Let $\rho$ be firm's belief probability that the market condition will be high in period $n$, then

$$
\rho=\rho_{i \mathrm{H}}=P\left(\Theta_{n}=\theta_{\mathrm{H}} \mid \Theta_{n-1}=\theta_{i}\right)
$$

when the realized market condition in the previous period is $\theta_{i}, i=\mathrm{H}, \mathrm{L}$.

Suppose the state of the system at the beginning of period $n$ is $(x, \rho)$. The firm reviews its inventory and offers a menu of contracts $s_{i}(x), i=\mathrm{H}, \mathrm{L}$, for period $n$ to maximize her total expected profit until the end of the planning horizon. Under this contract, the salesman's revenue for period $n$ is $s_{i}(x)=\alpha_{i}(x) D_{n}+\beta_{i}(x)$ when he signs contract $s_{i}$. As discussed before, we focus on the case that the salesperson is myopic and maximizes his expected utility for the current period, which is

$$
u\left(s_{i}, a_{i}\right)=-\mathbb{E}\left[e^{-\gamma\left[s_{i}\left(D_{n}\right)-a_{i}^{2} / 2\right]}\right] .
$$

The analysis of the salesman's problem is similar to that of the last period; the optimal effort level the salesman exerts is $\alpha_{\mathrm{H}}$ when the market condition is of type $\mathrm{H}$ and $\alpha_{\mathrm{L}}$ if the market condition is of type L. Hence, the certainty equivalence of the expected utility for the salesperson, if the market condition is of type $i=\mathrm{L}, \mathrm{H}$, is equal to

$$
\alpha_{i}(x)\left(\theta_{i}+\mu_{n}\right)+\alpha_{i}^{2}(x)\left(1-\gamma \sigma_{n}^{2}\right) / 2+\beta_{i}(x) .
$$

Now we consider the firm's problem of determining the optimal menu of compensation plans. By the revelation principle ([35]), it suffices for the firm to search for the optimal menu of contracts from the class of incentive compatible truth-telling contracts. Suppose the agent selects contract type $i$. As the replenishment decision is made after the sales agent selects the contract, the firm would know the market condition when placing the order. If the agent selects $\left(\alpha_{i}(x), \beta_{i}(x)\right)$, then the firm knows that the demand during the period is $D_{n}=\theta_{i}+\mu_{n}+\alpha_{i}(x)+\epsilon_{n}$, and hence, her operational decision is to replenish inventory in a way that maximizes her expected profit from periods $n$ to $N$. If the firm replenishes its inventory level to $y \geq x$, similar to (5), her current period expected profit is

$$
\begin{aligned}
c x & +\theta_{i}+\mu_{n}+\alpha_{i}(x)-\mathbb{E}\left[\alpha_{i}(x)\left(\theta_{i}+\mu_{n}+\alpha_{i}(x)+\epsilon_{n}\right)\right] \\
& -\beta_{i}(x)-(h+c) \mathbb{E}\left[\left(y-\theta_{i}-\mu_{n}-\alpha_{i}(x)-\epsilon_{n}\right)^{+}\right] \\
& -(p-c) \mathbb{E}\left[\left(\theta_{i}+\mu_{n}+\alpha_{i}(x)+\epsilon_{n}-y\right)^{+}\right] .
\end{aligned}
$$

As the market condition follows a two-state Markov chain, the market condition will be $\mathrm{H}$ type in period $n+1$ with probability $\rho_{i \mathrm{H}}$ and it will be L type with probability $\rho_{i \mathrm{~L}}$.
Hence, the maximum expected profit from period $n+1$ onward is

$$
\begin{aligned}
& \rho_{i \mathrm{H}} \mathbb{E}\left[V_{n+1}\left(\left(y-\theta_{i}-\mu_{n}-\alpha_{i}(x)-\epsilon_{n}\right)^{+}, \rho_{i \mathrm{H}}\right)\right] \\
& \quad+\rho_{i \mathrm{~L}} \mathbb{E}\left[V_{n+1}\left(\left(y-\theta_{i}-\mu_{n}-\alpha_{i}(x)-\epsilon_{n}\right)^{+}, \rho_{i \mathrm{~L}}\right)\right],
\end{aligned}
$$

recalling that $V_{n+1}(x, \rho)$ represents the firm's maximum expected profit from period $n+1$ onward when the inventory level is $x$ and her belief about the market condition being $\mathrm{H}$ is $\rho$.

Therefore, when contract $\left(\alpha_{i}(x), \beta_{i}(x)\right)$ is signed at the beginning of period $n$, the firm's optimization problem is to find the order-up-to level $y \geq x$ to maximize

$$
\begin{aligned}
c x & +\theta_{i}+\mu_{n}+\alpha_{i}(x)-\mathbb{E}\left[\alpha_{i}(x)\left(\theta_{i}+\mu_{n}+\alpha_{i}(x)-\epsilon_{n}\right)\right] \\
& -\beta_{i}(x)-(h+c) \mathbb{E}\left[\left(y-\theta_{i}+\mu_{n}-\alpha_{i}(x)-\epsilon_{n}\right)^{+}\right] \\
& -(p-c) \mathbb{E}\left[\left(\theta_{i}+\mu_{n}+\alpha_{i}(x)+\epsilon_{n}-y\right)^{+}\right] \\
& +\rho_{i \mathrm{H}} \mathbb{E}\left[V_{n+1}\left(\left(y-\theta_{i}-\mu_{n}-\alpha_{i}(x)-\epsilon_{n}\right)^{+}, \rho_{i \mathrm{H}}\right)\right] \\
& +\rho_{i \mathrm{~L}} \mathbb{E}\left[V_{n+1}\left(\left(y-\theta_{i}-\mu_{n}-\alpha_{i}(x)-\epsilon_{n}\right)^{+}, \rho_{i \mathrm{~L}}\right)\right] .
\end{aligned}
$$

For convenience, we let

$$
\begin{aligned}
W_{i}(y)= & -(h+c) \mathbb{E}\left[\left(y-\epsilon_{n}\right)^{+}\right]-(p-c) \mathbb{E}\left[\left(\epsilon_{n}-y\right)^{+}\right] \\
& +\rho_{i \mathrm{H}} \mathbb{E}\left[V_{n+1}\left(\left(y-\epsilon_{n}\right)^{+}, \rho_{i \mathrm{H}}\right)\right] \\
& +\rho_{i \mathrm{~L}} \mathbb{E}\left[V_{n+1}\left(\left(y-\epsilon_{n}\right)^{+}, \rho_{i \mathrm{~L}}\right)\right] .
\end{aligned}
$$

Note that, if $V_{n+1}(x, \rho)-c x$ is decreasing concave, then $W_{i}(y)$ is concave. To see this, note that

$$
\begin{aligned}
W_{i}(y)= & -h \mathbb{E}\left[\left(y-\epsilon_{n}\right)^{+}\right]-(p-c) \mathbb{E}\left[\left(\epsilon_{n}-y\right)^{+}\right] \\
& +\rho_{i H} \mathbb{E}\left[\left(V_{n+1}\left(\left(y-\epsilon_{n}\right)^{+}, \rho_{i H}\right)-c\left(y-\epsilon_{n}\right)^{+}\right)\right] \\
& +\rho_{i L} \mathbb{E}\left[\left(V_{n+1}\left(\left(y-\epsilon_{n}\right)^{+}, \rho_{i L}\right)-c\left(y-\epsilon_{n}\right)^{+}\right)\right] .
\end{aligned}
$$

Since $f(g(x))$ is concave when $f$ is decreasing concave and $g$ is convex, it follows from $V_{n+1}$ being decreasing concave, and $\left(y-\epsilon_{n}\right)^{+}$and $\left(\epsilon_{n}-y\right)^{+}$being convex, that $W_{i}(x)$ is concave. Let $q_{i}^{*}$ be the maximizer of $W_{i}(x)$.

We have the following result that characterizes the optimal production/inventory control policy of the firm as a marketbased state-dependent policy for an arbitrary period $n$.

PROPOSITION 2: (Market-Based State-Dependent Policy) The optimal inventory replenishing policy of the firm for an arbitrary period $n$ is a market-based state-dependent policy: if contract $\mathrm{s}_{i}(i=\mathrm{H}, \mathrm{L})$ is signed (and hence the market is of type $i$ ), then the optimal order-up-to level is $q_{i}^{*}+\theta_{i}+\mu_{n}+\alpha_{i}(x)$. That is, it is optimal to order $q_{i}^{*}-x+\theta_{i}+\mu_{n}+\alpha_{i}(x)$ if $x-\theta_{i}-\mu_{n}-\alpha_{i}(x) \leq q_{i}^{*}$ and order nothing otherwise.

This result shows that the effective information acquisition from the agent allows the firm to implement traditional inventory control strategies: the optimal inventory/production control policy is in general characterized by an order-up-to level 
that depends on (a) market condition and (b) the current inventory level [as $\alpha_{i}(x)$ is not constant in general]. Moreover, the following corollary shows that, although the incentive design problem and that of inventory control are highly interconnected, the optimal operational policy for periods with low inventory levels is interestingly the traditional simple base-stock policy, except that the base-stock level of a period depends on the extracted information from the agent about the market condition. However, it should be noted that the base-stock levels of two periods with the same extracted information (market condition) may be different due to effects such as seasonality.

COROLLARY 4: (Market-Based Base-Stock PolicyLow Inventory Periods) In periods with low inventory levels $\left(x \leq q_{i}^{*}+\theta_{i}+\mu_{n}+(1+\delta \mathbb{1}\{i=\mathrm{L}\}) /\left(1+\gamma \sigma_{n}^{2}\right)\right)$, the optimal production/inventory control policy is a market-based base-stock policy with base-stock level $q_{i}^{*}+\theta_{i}+\mu_{n}+(1+$ $\delta \mathbb{1}\{i=\mathrm{L}\}) /\left(1+\gamma \sigma_{n}^{2}\right)$.

The corollary above shows that, when the inventory level is sufficiently low, the commissions offered to both types of agents become inventory independent. Hence, demand becomes exogenously known (i.e., independent of the production decision). In such a situation, the firm first learns of the market condition from the agent (through contract design) and then follows the traditional base-stock policy, where the base-stock level is independent of on-hand inventory due to the exogenously known demand distribution.

Using Proposition 2, the optimal value function of (20) is

$$
\begin{aligned}
& c x+\theta_{i}+\mu_{n}+\alpha_{i}(x)-\mathbb{E}\left[\alpha_{i}(x)\left(\theta_{i}+\alpha_{i}(x)+\mu_{n}-\epsilon_{n}\right)\right] \\
& -\beta_{i}(x)+W_{i}\left(q_{i}^{*} \vee\left(x-\theta_{i}-\mu_{n}-\alpha_{i}(x)\right)\right) .
\end{aligned}
$$

Hence, using notation $\rho=\rho_{i \mathrm{H}}$, the firm's problem at the beginning of period $n$ is to solve

$$
\begin{aligned}
V_{n}(x, \rho)= & \max _{\alpha_{\mathrm{L}}, \alpha_{\mathrm{H}}, \beta_{\mathrm{H}}, \beta_{\mathrm{H}}}\left\{c x+\rho\left[\left(1-\alpha_{\mathrm{H}}(x)\right)\left[\theta_{\mathrm{H}}+\mu_{n}+\alpha_{\mathrm{H}}(x)\right]\right.\right. \\
& \left.-\beta_{\mathrm{H}}(x)+W_{\mathrm{H}}\left(q_{\mathrm{H}}^{*} \vee\left(x-\theta_{\mathrm{H}}-\mu_{n}-\alpha_{\mathrm{H}}(x)\right)\right)\right] \\
& +(1-\rho)\left[\left(1-\alpha_{\mathrm{L}}(x)\right)\left[\theta_{\mathrm{L}}+\mu_{n}+\alpha_{\mathrm{L}}(x)\right)\right] \\
& \left.\left.-\beta_{\mathrm{L}}(x)+W_{\mathrm{L}}\left(q_{\mathrm{L}}^{*} \vee\left(x-\theta_{\mathrm{L}}-\mu_{n}-\alpha_{\mathrm{L}}(x)\right)\right)\right]\right\},
\end{aligned}
$$

subject to IC, IR, and nonnegativity constraints similar to (8)-(12).

Moreover, similar to the analysis of the previous section, it can be seen that the firm's optimization problem can be reduced to, writing $\alpha_{i}(x)$ as $\alpha_{i}$,

$$
\begin{aligned}
& V_{n}(x, \rho)=\max _{\alpha_{\mathrm{H}}, \alpha_{\mathrm{L}}: \alpha_{\mathrm{H}} \geq \alpha_{\mathrm{L}} \geq 0}\left\{c x+\frac{\ln U_{0}}{\gamma}+\bar{\theta}+\mu_{n}\right. \\
& +\rho\left[\alpha_{\mathrm{H}}-\frac{1+\gamma \sigma^{2}}{2} \alpha_{\mathrm{H}}^{2}+W_{\mathrm{H}}\left(q_{\mathrm{H}}^{*} \vee\left(x-\theta_{\mathrm{H}}-\mu_{n}-\alpha_{\mathrm{H}}\right)\right)\right]
\end{aligned}
$$

Naval Research Logistics DOI 10.1002/nav

$$
\begin{aligned}
& +(1-\rho)\left[\alpha_{\mathrm{L}}-\frac{\rho}{1-\rho} \alpha_{\mathrm{L}}\left(\theta_{\mathrm{H}}-\theta_{\mathrm{L}}\right)-\frac{1+\gamma \sigma^{2}}{2} \alpha_{\mathrm{L}}^{2}\right. \\
& \left.\left.+W_{\mathrm{L}}\left(q_{\mathrm{L}}^{*} \vee\left(x-\theta_{\mathrm{L}}-\mu_{n}-\alpha_{\mathrm{L}}\right)\right)\right]\right\} .
\end{aligned}
$$

The following result shows that, similar to the last period, inventory has a (bounded from above) diminishing rate of return effect on the firm's profit.

PROPOSITION 3: (Concave Value Function-Arbitrary Period) For any $n, V_{n}(x, \rho)$ is concave in $x$ for any $\rho$, and $V_{n}(x, \rho)-c x$ is decreasing in $x$ for any $\rho$.

To find the optimal menu of contracts, we first solve the following two separate optimization problems:

$\max _{\alpha_{\mathrm{H}} \geq 0}\left\{\alpha_{\mathrm{H}}-\frac{1+\gamma \sigma_{n}^{2}}{2} \alpha_{\mathrm{H}}^{2}+W_{\mathrm{H}}\left(q_{\mathrm{H}}^{*} \vee\left(x-\theta_{\mathrm{H}}-\mu_{n}-\alpha_{\mathrm{H}}\right)\right\}\right.$,

and

$$
\begin{gathered}
\max _{\alpha_{\mathrm{L}} \geq 0}\left\{\alpha_{\mathrm{L}}-\frac{\rho}{1-\rho} \alpha_{\mathrm{L}}\left(\theta_{\mathrm{H}}-\theta_{\mathrm{L}}\right)-\frac{1+\gamma \sigma^{2}}{2} \alpha_{\mathrm{L}}^{2}\right. \\
\left.+W_{\mathrm{L}}\left(q_{\mathrm{L}}^{*} \vee\left(x-\theta_{\mathrm{L}}-\mu_{n}-\alpha_{\mathrm{L}}\right)\right)\right\} .
\end{gathered}
$$

It can be easily shown that, when $x \leq q_{\mathrm{H}}^{*}+\mu_{n}+\theta_{\mathrm{H}}+1 /(1+$ $\left.\gamma \sigma_{n}^{2}\right)$, the optimal solution of (22) is $\alpha_{\mathrm{H}}^{*}(x)=\left(1+\gamma \sigma_{n}^{2}\right)^{-1}$. If $x \geq q_{\mathrm{H}}^{*}+\mu_{n}+\theta_{\mathrm{H}}+1 /\left(1+\gamma \sigma^{2}\right)$, we let $\tilde{\alpha}_{\mathrm{H}}(x)$ be the solution to

$$
1-\left(1+\gamma \sigma_{n}^{2}\right) \alpha_{\mathrm{H}}-W_{\mathrm{H}}^{\prime}\left(x-\theta_{\mathrm{H}}-\mu_{n}-\alpha_{\mathrm{H}}\right)=0,
$$

which is increasing in $x$. It can also be seen that on $x \geq$ $q_{\mathrm{H}}^{*}+\mu_{n}+\theta_{\mathrm{H}}+1 /\left(1+\gamma \sigma^{2}\right)$, the optimal solution of (22) is $\alpha_{\mathrm{H}}^{*}(x)=\tilde{\alpha}_{\mathrm{H}}(x) \geq 1 /\left(1+\gamma \sigma_{n}^{2}\right)$. Similarly, for optimization problem (23), if $x \leq q_{\mathrm{L}}^{*}+\theta_{\mathrm{L}}+\mu_{n}+\delta\left(1+\gamma \sigma^{2}\right)^{-1}$ where, as before, $\delta=\left[1-\frac{\rho}{1-\rho}\left(\theta_{\mathrm{H}}-\theta_{\mathrm{L}}\right)\right]^{+}$, then the optimal solution of (23) is $\delta\left(1+\gamma \sigma_{n}^{2}\right)^{-1}$. If, however, $x \geq$ $q_{\mathrm{L}}^{*}+\theta_{\mathrm{L}}+\mu_{n}+\delta\left(1+\gamma \sigma^{2}\right)^{-1}$, we let $\tilde{\alpha}_{\mathrm{L}}(x)$ be the maximizer of

$$
\begin{aligned}
\alpha_{\mathrm{L}} & -\frac{\rho}{1-\rho} \alpha_{\mathrm{L}}\left(\theta_{\mathrm{H}}-\theta_{\mathrm{L}}\right)-\frac{1+\gamma \sigma^{2}}{2} \alpha_{\mathrm{L}}^{2} \\
& +W_{\mathrm{L}}\left(x-\theta_{\mathrm{L}}-\mu_{n}-\alpha_{\mathrm{L}}\right),
\end{aligned}
$$

which is also increasing in $x$. Then the optimal solution of (23) satisfies $\alpha_{\mathrm{L}}^{*}(x)=\tilde{\alpha}_{\mathrm{L}}(x) \geq \frac{\delta}{1+\gamma \sigma_{n}^{2}}$. The following lemma describes the structure of $\tilde{\alpha}_{\mathrm{H}}(x)$ and $\tilde{\alpha}_{\mathrm{L}}(x)$ for a specific range of interest where the firm is facing high levels of over-stock inventories. 
LEMMA 5 (High Over-Stock Inventories): For $x \geq$ $\max \left\{q_{\mathrm{H}}^{*}, q_{\mathrm{L}}^{*}\right\}+\theta_{\mathrm{H}}+\mu_{n}+\left(1+\gamma \sigma_{n}^{2}\right)^{-1}$, we have

a. $\tilde{\alpha}_{\mathrm{H}}(x)$ and $\tilde{\alpha}_{\mathrm{L}}(x)$ are increasing in $x$.

b. $\tilde{\alpha}_{\mathrm{H}}(x)$ and $\tilde{\alpha}_{\mathrm{L}}(x)$ converge to finite numbers as $x \rightarrow$ $\infty$, and $\lim _{x \rightarrow \infty} \tilde{\alpha}_{\mathrm{L}}(x)<\lim _{x \rightarrow \infty} \tilde{\alpha}_{\mathrm{H}}(x)$.

Unlike the analysis of the last period, we cannot prove that $\tilde{\alpha}_{\mathrm{H}}(x)$ and $\tilde{\alpha}_{\mathrm{L}}(x)$ are always concave for an arbitrary period. The above result, however, implies that (1) regardless of the agent type, the firm offers a more attractive commission rate when facing a higher inventory level and (2) the commission rate offered to the high type agent is more attractive than the one offered to the low type agent.

The following result characterizes the optimal menu of inventory-based contracts for an arbitrary period $n<N$. As expected, the optimal contracts are more complex than the last period. For the ease of notation, similar to the analysis of the last period, we define the following inventory intervals:

$$
\begin{aligned}
I_{1}= & \left(-\infty, q_{\mathrm{L}}^{*}+\theta_{\mathrm{L}}+\mu_{n}+\delta /\left(1+\gamma \sigma_{n}^{2}\right)\right], \\
I_{2}= & \left(q_{\mathrm{L}}^{*}+\theta_{\mathrm{L}}+\mu_{n}+\delta /\left(1+\gamma \sigma_{n}^{2}\right), z_{1}^{*}\right], \\
I_{3}= & \left(z_{1}^{*}, z_{2}^{*}\right], I_{4}=\left(z_{2}^{*}, z_{3}^{*}\right], I_{5}=\left(z_{3}^{*}, \infty\right), I_{1}^{\prime}=I_{1}, \\
I_{2}^{\prime}= & \left(q_{\mathrm{L}}^{*}+\theta_{\mathrm{L}}+\mu_{n}+\delta /\left(1+\gamma \sigma_{n}^{2}\right),\right. \\
& \left.q_{\mathrm{H}}^{*}+\theta_{\mathrm{H}}+\mu_{n}+1 /\left(1+\gamma \sigma_{n}^{2}\right)\right], \\
I_{3}^{\prime}= & \left(q_{\mathrm{H}}^{*}+\theta_{\mathrm{H}}+\mu_{n}+1 /\left(1+\gamma \sigma_{n}^{2}\right), z_{2}^{\prime *}\right], \\
I_{4}^{\prime}= & \left(z_{2}^{*}, z_{3}^{*}\right], I_{5}^{\prime}=\left(z_{3}^{*}, \infty\right), \\
I_{1}^{\prime \prime}= & \left(-\infty, q_{\mathrm{H}}^{*}+\theta_{\mathrm{H}}+\mu_{n}+1 /\left(1+\gamma \sigma_{n}^{2}\right)\right], \\
I_{2}^{\prime \prime}= & \left(q_{\mathrm{H}}^{*}+\theta_{\mathrm{H}}+\mu_{n}+1 /\left(1+\gamma \sigma_{n}^{2}\right),\right. \\
& \left.q_{\mathrm{L}}^{*}+\theta_{\mathrm{L}}+\mu_{n}+\delta /\left(1+\gamma \sigma_{n}^{2}\right)\right], \\
I_{3}^{\prime \prime}= & \left(q_{\mathrm{L}}^{*}+\theta_{\mathrm{L}}+\mu_{n}+\delta /\left(1+\gamma \sigma_{n}^{2}\right), z_{2}^{*}\right], \\
I_{4}^{\prime \prime}= & \left(z_{2}^{*^{\prime}}, z_{3}^{*^{\prime}}\right], I_{5}^{\prime \prime}=\left(z_{3}^{* \prime}, \infty\right),
\end{aligned}
$$

where $z_{1}^{*} \in\left(q_{\mathrm{L}}^{*}+\theta_{\mathrm{L}}+\mu_{n}+\delta /\left(1+\gamma \sigma_{n}^{2}\right), q_{\mathrm{H}}^{*}+\theta_{\mathrm{H}}+\mu_{n}+\right.$ $\left.1 /\left(1+\gamma \sigma_{n}^{2}\right)\right]$ is the solution to $\tilde{\alpha}_{\mathrm{L}}\left(z_{1}\right)=1 /\left(1+\gamma \sigma_{n}^{2}\right), z_{2}^{*}$ satisfies $z_{1}^{*} \leq z_{2}^{*}<q_{\mathrm{H}}^{*}+\theta_{\mathrm{H}}+\mu_{n}+1 /\left(1+\gamma \sigma_{n}^{2}\right)$ and is the solution to $z_{2}-\theta_{\mathrm{L}}-\mu_{n}-\alpha_{I_{3}}^{*}\left(z_{2}\right)=q_{\mathrm{L}}^{*}$ where $\alpha_{I_{3}}^{*}(x)$ is the maximizer of

$$
\begin{aligned}
& \alpha-\frac{1}{1+\gamma \sigma_{n}^{2}} \alpha^{2}-\rho\left(\theta_{\mathrm{H}}-\theta_{\mathrm{L}}\right) \alpha \\
& -(1-\rho) W_{\mathrm{L}}\left(x-\theta_{\mathrm{L}}-\mu_{n}-\alpha\right),
\end{aligned}
$$

$z_{3}^{*} \geq q_{\mathrm{H}}^{*}+\theta_{\mathrm{H}}+\mu_{n}+1 /\left(1+\gamma \sigma_{n}^{2}\right)$ is the greatest solution to $\tilde{\alpha}_{\mathrm{H}}\left(z_{3}\right)=\tilde{\alpha}_{\mathrm{L}}\left(z_{3}\right)$, and $z_{2}^{*}$ and $z_{3}^{*}$ are defined as the least and greatest points $z$ greater than $q_{\mathrm{L}}^{*}+\theta_{\mathrm{L}}+\mu_{n}+\delta /\left(1+\gamma \sigma_{n}^{2}\right)$ with $\tilde{\alpha}_{\mathrm{L}}(z)=\tilde{\alpha}_{\mathrm{H}}(z)$.
THEOREM 2 (Optimal Commissions-Arbitrary Period): The optimal menu of inventory-dependent contracts for period $n<N$ is determined by the two numbers $q_{\mathrm{H}}^{*}$ and $q_{\mathrm{L}}^{*}$. If $q_{\mathrm{H}}^{*}+\theta_{\mathrm{H}}+1 /\left(1+\gamma \sigma_{n}^{2}\right)>q_{\mathrm{L}}^{*}+\theta_{\mathrm{L}}+\delta /\left(1+\gamma \sigma_{n}^{2}\right)$, then the optimal commissions $\alpha_{\mathrm{H}}^{*}(x)$ and $\alpha_{\mathrm{L}}^{*}(x)$ are characterized based on two cases as described below.

CASE 1: $\tilde{\alpha}_{\mathrm{L}}\left(q_{\mathrm{H}}^{*}+\theta_{\mathrm{H}}+\mu_{n}+\frac{1}{1+\gamma \sigma_{n}^{2}}\right)>1 /\left(1+\gamma \sigma_{n}^{2}\right)$. In this case, the optimal commissions are as follows:

1. If $x \in I_{1}$, then $\alpha_{\mathrm{H}}^{*}(x)=\left(1+\gamma \sigma_{n}^{2}\right)^{-1}$ and $\alpha_{\mathrm{L}}^{*}(x)=$ $\delta\left(1+\gamma \sigma_{n}^{2}\right)^{-1}$;

2. if $x \in I_{2}$, then $\alpha_{\mathrm{H}}^{*}(x)=\left(1+\gamma \sigma_{n}^{2}\right)^{-1}$ and $\alpha_{\mathrm{L}}^{*}(x)=$ $\tilde{\alpha}_{\mathrm{L}}(x)$

3. if $x \in I_{3}$, then $\alpha_{\mathrm{H}}^{*}(x)=\alpha_{I_{3}}^{*}(x)_{+}$and $\alpha_{\mathrm{L}}^{*}(x)=$ $\alpha_{I_{3}}^{*}(x)_{-} ;$

4. if $x \in I_{4}$, then the optimal contract depends on whether $\tilde{\alpha}_{\mathrm{L}}(x)<\tilde{\alpha}_{\mathrm{H}}(x)$ or $\tilde{\alpha}_{\mathrm{L}}(x) \geq \tilde{\alpha}_{\mathrm{H}}(x)$; in the former case, $\alpha_{\mathrm{H}}^{*}(x)=\tilde{\alpha}_{\mathrm{H}}(x)$ and $\alpha_{\mathrm{L}}^{*}(x)=\tilde{\alpha}_{\mathrm{L}}(x)$, and in the latter case, $\alpha_{\mathrm{H}}^{*}(x)=\alpha_{I_{4}}^{*}(x)_{+}$and $\alpha_{\mathrm{L}}^{*}(x)=$ $\alpha_{I_{4}}^{*}(x)_{-}$, where $\alpha_{I_{4}}^{*}(x)$ is the optimizer of

$$
\begin{aligned}
\alpha- & \frac{1+\gamma \sigma_{n}^{2}}{2} \alpha^{2}+\rho \alpha\left(\theta_{\mathrm{H}}-\theta_{\mathrm{L}}\right) \\
& +\rho W_{\mathrm{H}}\left(x-\mu_{n}-\theta_{\mathrm{H}}-\alpha\right) \\
& +(1-\rho) W_{\mathrm{L}}\left(x-\mu_{n}-\theta_{\mathrm{L}}-\alpha\right) ;
\end{aligned}
$$

5. if $x \in I_{5}$, then $\alpha_{\mathrm{H}}^{*}(x)=\tilde{\alpha}_{\mathrm{H}}(x)$ and $\alpha_{\mathrm{L}}^{*}(x)=\tilde{\alpha}_{\mathrm{L}}(x)$.

CASE 2: $\tilde{\alpha}_{\mathrm{L}}\left(q_{\mathrm{H}}^{*}+\theta_{\mathrm{H}}+\mu_{n}+\frac{1}{1+\gamma \sigma_{n}^{2}}\right) \leq 1 /\left(1+\gamma \sigma_{n}^{2}\right)$. In this case, the optimal commissions are as follows:

1. If $x \in I_{1}^{\prime}$ then $\alpha_{\mathrm{H}}^{*}(x)=\left(1+\gamma \sigma_{n}^{2}\right)^{-1}$ and $\alpha_{\mathrm{L}}^{*}(x)=$ $\delta\left(1+\gamma \sigma_{n}^{2}\right)^{-1} ;$

2. if $x \in I_{2}^{\prime}$ then $\alpha_{\mathrm{H}}^{*}(x)=\left(1+\gamma \sigma_{n}^{2}\right)^{-1}$ and $\alpha_{\mathrm{L}}^{*}(x)=$ $\tilde{\alpha}_{\mathrm{L}}(x)$

3. if $x \in I_{3}^{\prime}$ then $\alpha_{\mathrm{L}}^{*}(x)=\tilde{\alpha}_{\mathrm{L}}(x)$ and $\alpha_{\mathrm{H}}^{*}(x)=\tilde{\alpha}_{\mathrm{H}}(x)$;

4. if $x \in I_{4}^{\prime}$, then the optimal contract depends on whether $\tilde{\alpha}_{\mathrm{L}}(x)<\tilde{\alpha}_{\mathrm{H}}(x)$ or $\tilde{\alpha}_{\mathrm{L}}(x) \geq \tilde{\alpha}_{\mathrm{H}}(x)$; in the former case, $\alpha_{\mathrm{L}}^{*}(x)=\tilde{\alpha}_{\mathrm{L}}(x)$ and $\alpha_{H}^{*}(x)=\tilde{\alpha}_{H}(x)$, and in the latter case, $\alpha_{\mathrm{H}}^{*}(x)=\alpha_{I_{4}}^{*}(x)_{+}$and $\alpha_{\mathrm{L}}^{*}(x)=$ $\alpha_{I_{4}}^{*}(x)_{-}$, where $\alpha_{I_{4}}^{*}(x)$ is the optimizer of (25); and

5. if $x \in I_{5}^{\prime}$, then $\alpha_{\mathrm{L}}^{*}(x)=\tilde{\alpha}_{\mathrm{L}}(z)$ and $\alpha_{\mathrm{H}}^{*}(x)=\tilde{\alpha}_{\mathrm{H}}(x)$.

However, if $q_{\mathrm{H}}^{*}+\theta_{\mathrm{H}}+1 /\left(1+\gamma \sigma_{n}^{2}\right) \leq q_{\mathrm{L}}^{*}+\theta_{\mathrm{L}}+\delta /\left(1+\gamma \sigma_{n}^{2}\right)$, then the optimal commissions are as follows:

1. If $x \in I_{1}^{\prime \prime}, \alpha_{\mathrm{H}}^{*}(x)=\left(1+\gamma \sigma_{n}^{2}\right)^{-1}$ and $\alpha_{\mathrm{L}}^{*}(x)=$ $\delta\left(1+\gamma \sigma_{n}^{2}\right)^{-1}$;

2. if $x \in I_{2}^{\prime \prime}$, then $\alpha_{\mathrm{H}}^{*}(x)=\tilde{\alpha}_{\mathrm{H}}(x)$ and $\alpha_{\mathrm{L}}^{*}(x)=$ $\delta\left(1+\gamma \sigma_{n}^{2}\right)^{-1}$;

Naval Research Logistics DOI 10.1002/nav 
3. if $x \in I_{3}^{\prime \prime}$, then $\alpha_{\mathrm{H}}^{*}(x)=\tilde{\alpha}_{\mathrm{H}}(x)$ and $\alpha_{\mathrm{L}}^{*}(x)=\tilde{\alpha}_{\mathrm{L}}(x)$;

4. if $x \in I_{4}^{\prime \prime}$, then the optimal contract depends on whether $\tilde{\alpha}_{\mathrm{L}}(x)<\alpha_{\mathrm{H}}(x)$ or $\tilde{\alpha}_{\mathrm{L}}(x) \geq \alpha_{\mathrm{H}}(x)$; in the former case, $\alpha_{\mathrm{H}}^{*}(x)=\tilde{\alpha}_{\mathrm{H}}(x)$ and $\alpha_{L}^{*}(x)=\tilde{\alpha}_{L}(x)$, and in the latter case, $\alpha_{\mathrm{H}}^{*}(x)=\alpha_{I_{4}}^{*}(x)_{+}$and $\alpha_{\mathrm{L}}^{*}(x)=$ $\alpha_{I_{4}}^{*}(x)_{-}$, where $\alpha_{I_{4}}^{*}(x)$ is the optimizer of (25); and

5. if $x \in I_{5}^{\prime \prime}$, then $\alpha_{\mathrm{H}}^{*}(x)=\tilde{\alpha}_{\mathrm{H}}(x)$ and $\alpha_{L}^{*}(x)=\tilde{\alpha}_{L}(x)$.

From the above theorem, it can be seen that the optimal commissions offered by the firm in each period are typically (1) increasing in the firm's inventory level, (2) decreasing in the agent's risk aversion level, and (3) concave in each inventory range. Moreover, it can be seen that if $\delta=0$, then the lower type agent is only paid a fixed/salary payment during low inventory periods. Also, combining Theorem 2 with Proposition 2 implies that, the demand in our setting is stochastically increasing in the inventory level, and the firm's ordering quantity is decreasing in its inventory level in each inventory range.

\subsection{A Heuristic Menu of Inventory-Based Compensation Plans}

The optimal menu of contracts for an arbitrary period $n$ characterized in Theorem 2 is rather complex and not easy to implement. Hence, we now propose a much simpler but still effective heuristic menu of contracts.

To develop a heuristic menu of contracts, we benefit from the analysis of the last period presented in Section 4. More specifically, we show how the structure of the optimal contracts in the last period can be used to generate a simple and implementable menu of inventory-dependent contracts for an arbitrary period. Thus, the firm can benefit from such a menu of contracts along with the market-based inventory control policy identified in Proposition 2 to effectively increase its profit.

Our proposed heuristic menu of contracts is as follows. In each period $n$, we first compute $q_{i}^{*}$ (for $i=\mathrm{H}, \mathrm{L}$ ). Next, if it holds that $q_{\mathrm{H}}^{*}+\theta_{\mathrm{H}}+1 /\left(1+\gamma \sigma_{n}^{2}\right) \leq q_{\mathrm{L}}^{*}+\theta_{\mathrm{L}}+\delta /\left(1+\gamma \sigma_{n}^{2}\right)$, then the contracts are the same as those presented in Theorem 2 , but with $z_{2}^{*}=z_{3}^{*}=q_{\mathrm{L}}^{*}+\theta_{\mathrm{L}}+\delta /\left(1+\gamma \sigma_{n}^{2}\right)$ (i.e., assuming $\left.I_{4}^{\prime \prime}=\emptyset\right)$. Hence, the contract structure is extremely simple in this case and commission for type $i$ agent is either constant or equal to $\tilde{\alpha}_{i}(x)$. If $q_{\mathrm{H}}^{*}+\theta_{\mathrm{H}}+1 /\left(1+\gamma \sigma_{n}^{2}\right)>$ $q_{\mathrm{L}}^{*}+\theta_{\mathrm{L}}+\delta /\left(1+\gamma \sigma_{n}^{2}\right)$, then the contract is similar to that of the last period presented in Theorem 1. Specifically, we first check whether Case 1 or Case 2 of Theorem 2 holds. If Case 1 (Case 2) holds, then the contract is similar to Part (1) (Part (2) of Theorem 1 , but with $\mu$ and $\sigma^{2}$ replaced with with $\mu_{n}$ and $\sigma_{n}^{2}$, respectively.

We now investigate the performance of the proposed heuristic menu of contracts. To this end, we compare it with (1) the optimal and (2) the inventory-independent menu of contracts of [9]. This will shed light on the performance of the proposed heuristic, and also on the importance of considering dynamic inventory levels when designing compensation plans: a fact that has been largely overlooked in the literature.

Numerical Study 2. We consider a problem with planning horizon of $N=3$, where $\mu_{1}=3$, and $\mu_{n}=\mu_{n-1}+\eta(n=2,3$ and $\eta=-1,-0.5,0,0.5,1), \sigma_{1}=0.5, \sigma_{2}=0.4, \sigma_{3}=0.3$, $\rho_{\mathrm{HH}}=0.6, \rho_{\mathrm{HL}}=0.3, \rho_{\mathrm{LH}}=0.3, \rho_{\mathrm{LL}}=0.7$, and the other parameters are the same as those in Numerical Study 1 (i.e., $p=7, h=1, c=2, \theta_{\mathrm{H}}=5, \theta_{\mathrm{L}}=1, \gamma=2$, and $\left.U_{0}=10\right)$. Assuming that at the beginning of the horizon, the firm does not have any on-hand inventory, and that the market condition is high, we first find the optimal expected profit of the firm for all of the five test cases parameterized by $\eta$ (which represents an increasing or decreasing trend in the market demand). To find the optimal expected profit, we solve the constrained optimal dynamic programming Eq. (21) after a discretization of the continuous inventory state space with a precision of 0.2 as well as a truncation to range $[-2,6]$ (i.e., considering a cardinality of 40 for the inventory space). Next, using the same discretization method, we compute the performance of the proposed inventory-dependent menu of contracts as well as the inventory-independent menu of contracts of [9] (i.e., with $\alpha_{\mathrm{H}}^{*}(x)=\left(1+\gamma \sigma_{n}^{2}\right)^{-1}$ and $\left.\alpha_{\mathrm{L}}^{*}(x)=\delta\left(1+\gamma \sigma_{n}^{2}\right)^{-1}\right)$ for all test cases.

The performance of the two heuristics compared to the optimal one is depicted in Fig. 2 using the percentage optimality gap (defined as the percentage difference between total profit under the heuristic strategy and that of the optimal strategy). From this figure, the proposed inventorydependent heuristic shows an average optimality gap of $1.69 \%$, while the inventory-independent heuristic shows an average optimal gap of $6.62 \%$ : incorporating the inventory

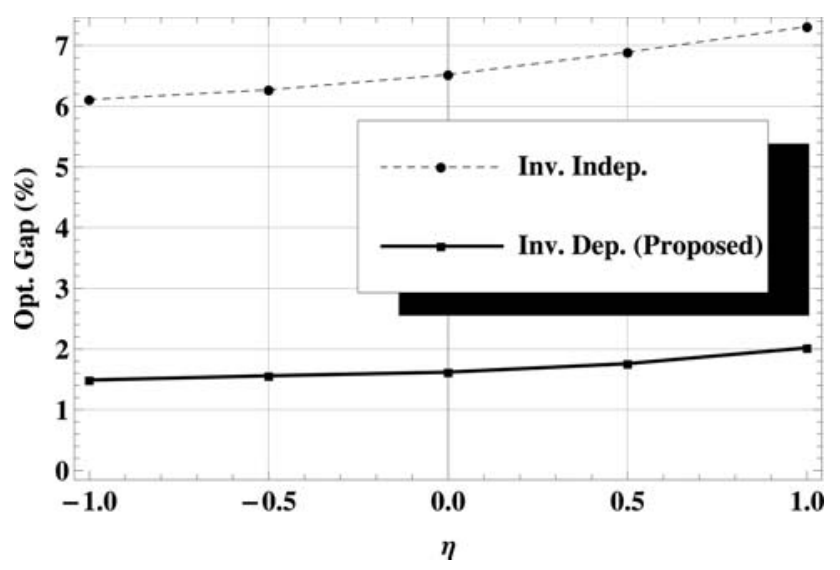

Figure 2. Performance of the proposed heuristic menu of contracts compared to (a) the optimal and (b) the inventory-independent policy for different test cases. Parameter $\eta$ represents an increase or decrease in the demand $\left(\mu_{n}=\mu_{n-1}+\eta\right)$. 
level in compensation design (through the proposed heuristic) reduces the average optimality gap by about $74 \%$. These results clearly illuminate the disadvantage of separating production/inventory control decision with that of salesforce incentive design as is currently widely done in both research and practice. Furthermore, from Fig. 2, we observe that both heuristic menus of contracts show a better performance when demand in future periods is decreasing. This is to some extent expected, as both heuristics are built upon characteristics of the optimal contracts in a single period; if future demand is negligible compared to the current demand, the greedy heuristics are expected to work well. Furthermore, we observe that the proposed inventory-dependent policy is more robust to changes in the future market demand compared to the inventory-independent heuristic. In fact, the optimality gap range (the difference between maximum and minimum optimality gap in the test suite) for the proposed policy and the inventory-independent heuristic are 0.53 and $1.20 \%$, respectively. This observation shows that the proposed heuristic is very effective in considering the current inventory level decisions for meeting the requirement of the future periods.

To gain deeper insights into the performance of the proposed heuristic menu of contracts, we conduct another numerical study and demonstrate the robustness of the heuristic policy with respect to variations in the starting stock level.

Numerical Study 3. Consider again the problem with planning horizon of $N=3$, and parameters $p=5, h=1$, $c=1.5, \theta_{\mathrm{H}}=3, \theta_{\mathrm{L}}=1, \gamma=2, U_{0}=5, \mu_{1}=\mu_{2}=\mu_{3}=$ $3, \sigma_{1}=0.5, \sigma_{2}=0.4, \sigma_{3}=0.3, \rho_{\mathrm{HH}}=0.5, \rho_{\mathrm{HL}}=0.5$, $\rho_{\mathrm{LH}}=0.3, \rho_{\mathrm{LL}}=0.7$. Assuming the market condition at the beginning of horizon is high, Fig. 3 compares the performance of both the proposed inventory-dependent heuristic and the inventory-independent contract (defined in Numerical Study 2) with that of the optimal for various starting inventory levels (i.e., the inventory level at the beginning of the planning horizon). From Fig. 3, we observe that the optimality gap for both heuristics in this example is lower than that of Numerical Study 2. However, the proposed inventorydependent heuristic still has a much lower average optimality gap $(0.43 \%)$ compared to the inventory-independent one $(3.43 \%)$. Furthermore, as is expected, the performance of the proposed heuristic becomes better (compared to the optimal policy) as the starting inventory increases, but the performance of the inventory-independent heuristic becomes worse. This is because (a) when the inventory level in each period is sufficiently high (the result of a very high starting inventory level), the proposed heuristic is essentially the same as the optimal one, and (b) the inventory-independent menu of contracts gets closer to the optimal for very low levels of inventory, and hence, is expected to perform its best when the inventory level in each period is low.

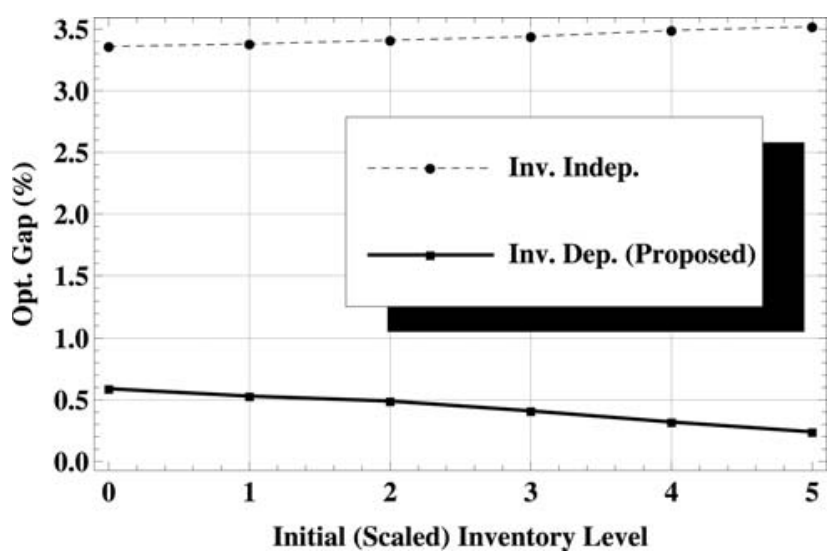

Figure 3. The effect of starting inventory level on the performance of the proposed heuristic menu of contracts compared to (a) the optimal and (b) the inventory-independent policy.

\section{CONCLUDING REMARKS}

In this article, we rigorously demonstrated the significant effect of inventory levels on the design of salesforce incentives. We provided several managerial insights through developing and analyzing a dynamic Principal-Agent model with both moral hazard and adverse selection. We showed that the optimal compensation offer is defined by a menu of contracts which highly depend on the inventory level. We completely characterized this menu of contracts, and found that the optimal commission rates offered by the firm are increasing in the inventory levels, describing the observed behavior of firms in practice. In addition, we found that the firm needs to offer higher commission rates to its less riskaverse agents. These analytical findings support the empirical studies that find (i) changing compensation plans is effective in increasing sales (e.g., [31]), and (ii) more risk-averse individuals prefer a fixed salary and are less productive under incentive pays (e.g., [6]).

In addition to illuminating the optimal design of the compensation packages when inventory levels are taken into account, we characterized the firm's optimal inventory/production control policy. We found that in general it can be characterized as a market-based state-dependent policy where the firm only needs to take advantage of the extracted information from the agent. Furthermore, making use of such information, the optimal inventory control policy translates to a simple traditional base-stock policy in periods with low inventory levels. These results show that information acquisition through contract design can enable firms to implement the classic inventory control policies.

For the ease of exposition and analysis, we made some modeling assumptions. Although they may seem restrictive, we note that the results and insights provided are robust to many of those modeling assumptions. For instance, the 
system parameters including $p, c, h$, agent's risk parameter $\gamma$, or agent's cost of effort $v(a)$ are assumed to be period independent. We point out that all the results can be extended to the case with period-dependent parameters. This extension is valid and useful in applications. For example, these parameters could be different for summer and winter seasons. Also, for tractability and consistency with the literature, we assumed that the market condition can only take two values. Analysis with more than two possible levels of market condition will be much more complex, but the main insights will not change.

We considered the case in competitive markets with many qualified agents in which contracts do not provide any longterm employment guarantee. In such an environment, the agent follows a roughly "myopic" behavior and tries to optimize gains in the current period. This allows us to apply the revelation principle in the design of the dynamic mechanism. That is, we were able to conveniently restrict our attention to the class of mechanisms in which the agent is truth-telling. In scenarios where the environment is not highly competitive, and the agent may become strategic, possibly disguising himself to sacrifice his utility for the current period to obtain higher utility for future periods. For instance, knowing that the commission rates will become more attractive if the inventory builds up, the agent may have some incentives not to work hard enough, or prefer to sign the contract that does not match his type. These can give out wrong signals to the firm and may mislead the firm's mechanism design, which makes the problem analytically intractable. While our framework may provide a tractable approximation, we note that the dynamic mechanism design problem with a strategic agent is a challenging but also a very interesting setting to study, which we leave for a careful future study (see also $[28,36,38])$.

There are some other interesting directions for future research. The first is to consider the scenario with multiple sales agents. In such a scenario, the firm needs to design a contract for each and every one of them. The terms of the contracts are usually different as agents are heterogeneous and have different risk attitudes. We note that the resulting optimization problem, after simplification, is similar to (15) except that the decisions are $\alpha$ 's and $\beta$ 's for all agents. Clearly, the optimal contracts for the agents are not independent due to inventory pooling, and the risk parameters for the different agents will be instrumental in finding the optimal compensation contracts.

Another interesting direction to consider is when pricing is also a decision. In that case, the demand will also depend on the selling price. We found that most of the results in the article can be carried over to the case of additive demand, in which the demand function is defined as $D_{n}=\Theta_{n}+a_{n}+\mu_{n}-d_{n}\left(p_{n}\right)+\epsilon_{n}$, where $d_{n}(n)$ is a decreasing function of selling price $p_{n}$ in period $n$. Under the common assumption that the revenue function $x d_{n}^{-1}(x)$ is concave, where $d_{n}^{-1}($.$) is the inverse function of d_{n}($.$) , similar$ inventory and pricing decisions as [19] can be obtained (similar to [10], the decision of inventory and pricing need to be transformed into decision of inventory and average demand), and the optimal contract is still the same as what we presented in Section 5. Furthermore, if pricing is a decision, it will be interesting to compare the case where the pricing decision is made by the firm with the case where the pricing decision is delegated to the sales agent. There has been plenty of research in the Marketing literature on this issue (see e.g., $[29,4$, or 34]), and it will be interesting to revisit the insights provided by such studies when operational decisions are also taken into account.

\section{APPENDIX: PROOFS}

PROOF OF LEMMA 1: (a) Note that on $x \geq q^{*}+\theta_{\mathrm{H}}+\mu+1 /\left(1+\gamma \sigma^{2}\right)$, $\alpha_{\mathrm{H}}(x)$ and $\alpha_{\mathrm{L}}(x)$ are, respectively, the solution to

$$
(p+h) \Phi\left(x-\theta_{\mathrm{H}}-\mu-\alpha\right)-\left(1+\gamma \sigma^{2}\right) \alpha-p+c+1=0,
$$

and

$$
\begin{aligned}
& (p+h) \Phi\left(x-\theta_{\mathrm{L}}-\mu-\alpha\right)-\left(1+\gamma \sigma^{2}\right) \alpha-p+c+1 \\
& -\frac{\rho}{1-\rho}\left(\theta_{\mathrm{H}}-\theta_{\mathrm{L}}\right)=0,
\end{aligned}
$$

Taking derivative with respect to $x$ yields

$$
\begin{aligned}
& \alpha_{\mathrm{H}}^{\prime}(x)=\frac{(p+h) \phi\left(x-\theta_{\mathrm{H}}-\mu-\alpha_{\mathrm{H}}(x)\right)}{(p+h) \phi\left(x-\theta_{\mathrm{H}}-\mu-\alpha_{\mathrm{H}}(x)\right)+\left(1+\gamma \sigma^{2}\right)}, \\
& \alpha_{\mathrm{L}}^{\prime}(x)=\frac{(p+h) \phi\left(x-\theta_{\mathrm{L}}-\mu-\alpha_{\mathrm{L}}(x)\right)}{(p+h) \phi\left(x-\theta_{\mathrm{L}}-\mu-\alpha_{\mathrm{L}}(x)\right)+\left(1+\gamma \sigma^{2}\right)}
\end{aligned}
$$

Thus, $0 \leq \alpha_{\mathrm{H}}^{\prime}(x) \leq 1,0 \leq \alpha_{\mathrm{L}}^{\prime}(x) \leq 1$. This proves that $\alpha_{\mathrm{H}}(x), \alpha_{\mathrm{L}}(x), x-$ $\alpha_{\mathrm{H}}(x)$ and $x-\alpha_{\mathrm{L}}(x)$ are all increasing functions.

(b) We only prove that $\alpha_{\mathrm{H}}(x)$ converges as $x \rightarrow \infty$, as similar analysis applies to $\alpha_{\mathrm{L}}(x)$. As $\alpha_{\mathrm{H}}(x)$ and $x-\alpha_{\mathrm{H}}(x)$ are both increasing, $\Phi\left(x-\theta_{\mathrm{H}}-\right.$ $\left.\mu-\alpha_{\mathrm{H}}(x)\right)$ converges to a nonnegative number less than or equal to 1 , and $\alpha_{\mathrm{H}}(x)$ also converges to a limit, possibly infinity. We show that this limit has to be finite. To see this, recall that by (26) and (27), $\theta_{\mathrm{H}}+\mu+\alpha_{\mathrm{H}}(x)$ is the solution $z$ to

$$
(p+h) \Phi(x-z)-\left(1+\gamma \sigma^{2}\right) z-p+c+1+\left(1+\gamma \sigma^{2}\right)\left(\theta_{\mathrm{H}}+\mu\right)=0,
$$

while $\theta_{\mathrm{L}}+\mu+\alpha_{\mathrm{L}}(x)$ is the solution $z$ to

$$
\begin{aligned}
& (p+h) \Phi(x-z)-\left(1+\gamma \sigma^{2}\right) z-p+c+1+\left(1+\gamma \sigma^{2}\right)\left(\theta_{\mathrm{L}}+\mu\right) \\
& \quad-\frac{\rho}{1+\rho}\left(\theta_{\mathrm{H}}-\theta_{\mathrm{L}}\right)=0 .
\end{aligned}
$$

It follows from (30) that

$$
(p+h) \Phi\left(x-\theta_{\mathrm{H}}-\mu-\alpha_{\mathrm{H}}(x)\right)-p+c+1=\left(1+\gamma \sigma^{2}\right) \alpha_{\mathrm{H}}(x) .
$$

As the left-hand side converges to a finite number, it follows that $\alpha_{\mathrm{H}}(x)$ also converges to a finite number as $x \rightarrow \infty$. 
Substituting $\theta_{\mathrm{H}}-\mu-\alpha_{\mathrm{H}}(x)$ and $\theta_{\mathrm{L}}-\mu-\alpha_{\mathrm{L}}(x)$ in (30) and (31), and subtracting the results, we obtain

$$
\begin{gathered}
(p+h)\left[\Phi\left(x-\theta_{\mathrm{H}}-\mu-\alpha_{\mathrm{H}}(x)\right)-\Phi\left(x-\theta_{\mathrm{L}}-\mu-\alpha_{\mathrm{L}}(x)\right)\right] \\
-\frac{\rho}{1-\rho}\left(\theta_{\mathrm{H}}-\theta_{\mathrm{L}}\right)=\left(1+\gamma \sigma^{2}\right)\left(\alpha_{\mathrm{H}}(x)-\alpha_{\mathrm{L}}(x)\right)
\end{gathered}
$$

As $\alpha_{\mathrm{H}}(x)$ and $\alpha_{\mathrm{L}}(x)$ converge to finite numbers, $x-\theta_{\mathrm{H}}-\mu-\alpha_{\mathrm{H}}(x)$ and $x-\theta_{\mathrm{L}}-\mu-\alpha_{\mathrm{L}}(x)$ both go to infinity as $x \rightarrow \infty$. Therefore, the first term on the left-hand side of (32) goes to 0 as $x \rightarrow \infty$. As the second term of the left-hand side of (32) is a negative constant, this shows that the right-hand side converges to $-\rho\left(\theta_{\mathrm{H}}-\theta_{\mathrm{L}}\right) /(1-\rho)<0$ as $x \rightarrow \infty$, implying

$$
\lim _{x \rightarrow \infty}\left\{\alpha_{\mathrm{H}}(x)-\alpha_{\mathrm{L}}(x)\right\}=-\frac{\frac{\rho}{1-\rho}\left(\theta_{\mathrm{H}}-\theta_{\mathrm{L}}\right)}{1+\gamma \sigma^{2}}<0,
$$

which proves part (b).

(c) Showing $\alpha_{\mathrm{H}}(x)$ is concave is equivalent to showing that $\alpha_{\mathrm{H}}^{\prime}(x)$ is decreasing. By (28), it suffices to prove that $\phi\left(x-\theta_{\mathrm{H}}-\mu-\alpha_{\mathrm{H}}(x)\right)$ is decreasing in $x$. From part (a), $x-\alpha_{\mathrm{H}}(x)$ is increasing in $x$. As $\phi(x)$ is strictly decreasing on $x \geq 0$, it suffices to prove that, in the range of consideration, we have $x-\theta_{\mathrm{H}}-\mu-\alpha_{\mathrm{H}}(x) \geq 0$.

From our analysis, the optimization of (16) is independent of $G($.$) and$ is given by $1 /\left(1+\gamma \sigma^{2}\right)$ on $x \leq q^{*}+\theta_{\mathrm{H}}+\mu+1 /\left(1+\gamma \sigma^{2}\right)$, while on $x \geq q^{*}+\theta_{\mathrm{H}}+\mu+1 /\left(1+\gamma \sigma^{2}\right)$, the optimizer of (16) satisfies $x-\theta_{\mathrm{H}}-\mu-\alpha_{\mathrm{H}}(x) \geq q^{*} \geq 0$. Therefore, $\alpha_{\mathrm{H}}(x)$ is concave in $x$ on $x \geq q^{*}+\theta_{\mathrm{H}}+\mu+1 /\left(1+\gamma \sigma^{2}\right)$.

Similar argument shows that $\alpha_{\mathrm{L}}(x)$ is concave on $x \geq \theta_{\mathrm{L}}+\mu+\delta /(1+$ $\left.\gamma \sigma^{2}\right)$, and that on this range, $x-\theta_{\mathrm{L}}-\mu-\alpha_{\mathrm{L}}(x) \geq q^{*} \geq 0$. In particular, these results are true on $x \geq q^{*}+\theta_{\mathrm{H}}+\mu+1 /\left(1+\gamma \sigma^{2}\right)$.

(d) We next prove $\alpha_{\mathrm{H}}(x)-\alpha_{\mathrm{L}}(x)$ is strictly increasing on $x \geq q^{*}+\theta_{\mathrm{H}}+$ $1 /\left(1+\gamma \sigma^{2}\right)$, or $\alpha_{\mathrm{H}}^{\prime}(x)>\alpha_{\mathrm{L}}^{\prime}(x)$. By (28) and (29) and that $\phi(x)$ is strictly decreasing on $x \geq 0$, it suffices to prove

$$
\theta_{\mathrm{H}}+\alpha_{\mathrm{H}}(x) \geq \theta_{\mathrm{L}}+\theta_{\mathrm{L}}(x)
$$

$x-\theta_{\mathrm{H}}-\mu-\alpha_{\mathrm{H}}(x) \geq 0$ and $x-\theta_{\mathrm{L}}-\mu-\alpha_{\mathrm{L}}(x) \geq 0$. The two latter inequalities are proved in part (c). Hence, in the following, we prove (33). To show (33), recall that $\theta_{\mathrm{H}}+\mu+\alpha_{\mathrm{H}}(x)$ is the solution to (30), while $\theta_{\mathrm{L}}+\mu+\alpha_{\mathrm{L}}(x)$ is the solution to (31). Because $(p+h) \Phi(x-z)-\left(1+\gamma \sigma^{2}\right) z$ is strictly decreasing in $z$, it follows from $\left(1+\gamma \sigma^{2}\right) \theta_{\mathrm{H}}>\left(1+\gamma \sigma^{2}\right) \theta_{\mathrm{L}}-\frac{\rho}{1+\rho}\left(\theta_{\mathrm{H}}-\theta_{\mathrm{L}}\right)$ that the solution to (30) is less than the solution to (31). This proves $\theta_{\mathrm{H}}+\alpha_{\mathrm{H}}(x)>\theta_{\mathrm{L}}+\alpha_{\mathrm{L}}(x)$, which completes the proof of part (c).

PROOF OF LEMMA 2: Recall that $q^{*}=\Phi^{-1}((p-c) /(p+h))$. As $\Phi$ is strictly increasing, $\theta>\xi(\rho, \theta)$ if, and only if, $\Phi\left(\theta+q^{*}\right)>\frac{p-c+\frac{\rho \theta}{1-\rho}}{p+h}$. Let $g(\theta)=\Phi\left(\theta+q^{*}\right)-\frac{p-c+\frac{\rho \theta}{1-\rho}}{p+h}$. Then, $g(0)=0$, and $\lim _{\theta \rightarrow \infty} g(\theta)=-\infty$. Since $q^{*} \geq 0, g^{\prime \prime}(\theta)=\phi^{\prime}\left(\theta+q^{*}\right)<0$ on $\theta \geq 0$. This shows that $g(\theta)$ is a concave function on $\theta \geq 0$. Hence, there exists a $\bar{\theta}$ such that $g(\bar{\theta})=0$ if, and only if, $g^{\prime}(0)>0$, or $\phi\left(q^{*}\right)>\frac{\rho}{1-\rho}$. This is the same as $\rho<\rho^{*}$. Thus, when $\rho<\rho^{*}$, we define $\bar{\theta}(\rho)>0$ as the solution to $g(\theta)=0$. Then on $0 \leq \theta \leq \bar{\theta}(\rho)$, we have $\theta>\xi(\rho, \theta)$, and $\theta \leq \xi(\rho, \theta)$ on $\theta \geq \bar{\theta}(\rho)$. However, if $\rho \geq \rho^{*}$, then $g^{\prime}(0) \leq 0$. Hence, $g(\theta) \leq 0$ for all $\theta \geq 0$. This shows that, when $\rho \geq \rho^{*}, \theta \leq \xi(\rho, \theta)$ for all $\theta \geq 0$.

PROOF OF LEMMA 3: By Lemma 2, if one of the conditions (a) or (b) is satisfied, we have $\theta_{\mathbf{H}}-\theta_{\mathrm{L}}<\xi\left(\rho, \theta_{\mathbf{H}}-\theta_{\mathrm{L}}\right)$, or

$$
q^{*}+\theta_{\mathrm{H}}-\theta_{\mathrm{L}}<\Phi^{-1}\left(\frac{p-c+\frac{\rho}{1-\rho}\left(\theta_{\mathrm{H}}-\theta_{\mathrm{L}}\right)}{p+h}\right) .
$$

Furthermore, by part (d) of Lemma 1, the necessary and sufficient condition for the existence of a finite number $z^{*}>q^{*}+\theta_{\mathrm{H}}+\mu+1 /\left(1+\gamma \sigma^{2}\right)$ such that $\alpha_{\mathrm{H}}(x)<\alpha_{\mathrm{L}}(x)$ on $z^{*}>x \geq q^{*}+\theta_{\mathrm{H}}+1 /\left(1+\gamma \sigma^{2}\right), \alpha_{\mathrm{H}}\left(z^{*}\right)=\alpha_{\mathrm{L}}\left(z^{*}\right)$, and $\alpha_{\mathrm{H}}(x)>\alpha_{\mathrm{L}}(x)$ on $x>z^{*}$, is

$$
\alpha_{\mathrm{L}}\left(q^{*}+\theta_{\mathrm{H}}+\mu+\frac{1}{1+\gamma \sigma^{2}}\right)>\frac{1}{1+\gamma \sigma^{2}} .
$$

Since on $x \geq q^{*}+\theta_{\mathrm{L}}+\mu+\delta /\left(1+\gamma \sigma^{2}\right), \theta_{\mathrm{L}}+\mu+\alpha_{\mathrm{L}}(x)$ is the solution of (31) and the left-hand side of (31) is decreasing in $z=\theta_{\mathrm{L}}+\mu+\alpha_{\mathrm{L}}$, (35) is satisfied if, and only if, when $x=q^{*}+\theta_{\mathrm{H}}+\mu+\frac{1}{1+\gamma \sigma^{2}}$, the left-hand side of (31) is positive with $z=\theta_{\mathrm{L}}+\mu+\frac{1}{1+\gamma \sigma^{2}}$. That is (35) is satisfied if, and only if,

$$
\begin{gathered}
(p+h) \Phi\left(q^{*}+\theta_{\mathrm{H}}+\mu+\frac{1}{1+\gamma \sigma^{2}}-z\right)-\left(1+\gamma \sigma^{2}\right) z-p+c+1 \\
+\left(1+\gamma \sigma^{2}\right)\left(\theta_{\mathrm{L}}+\mu\right)-\left.\frac{\rho}{1+\rho}\left(\theta_{\mathrm{H}}-\theta_{\mathrm{L}}\right)\right|_{z=\theta_{\mathrm{L}}+\mu+\frac{1}{1+\gamma \sigma^{2}}}>0 .
\end{gathered}
$$

Canceling common terms, this is equivalent to

$$
(p+h) \Phi\left(q^{*}+\theta_{\mathrm{H}}-\theta_{\mathrm{L}}\right)>p-c+\frac{\rho}{1-\rho}\left(\theta_{\mathrm{H}}-\theta_{\mathrm{L}}\right) .
$$

As $\Phi$ is strictly increasing, this is equivalent to (34), and the existence of $z^{*}$ is established.

Now suppose neither of conditions (a) or (b) of Lemma 3 holds. Then, by Lemma 2, the opposite direction of (34) is satisfied. But the same argument as above shows that $\alpha_{\mathrm{H}}(x) \geq \alpha_{\mathrm{L}}(x)$ on all $x \geq q^{*}+\theta_{\mathrm{H}}+\mu+1 /\left(1+\gamma \sigma^{2}\right)$ if, and only if, the opposite direction of (36), or equivalently, the opposite direction of (34) holds. This completes the proof.

PROOF OF THEOREM 1: Before proving this result, we note that the control parameters $z_{1}^{*}, z_{2}^{*}$, and $z_{3}^{*}$ used in defining inventory intervals $I_{2}, I_{3}, \ldots, I_{5}$ satisfy $z_{1}^{*}<z_{2}^{*}<q^{*}+\theta_{\mathrm{H}}+\mu+\frac{1}{1+\gamma \sigma^{2}}<z_{3}^{*}$. To see $z_{1}^{*}<z_{2}^{*}$, by the definition of $z_{2}^{*}$ and that $x-\tilde{\alpha}_{\mathrm{L}}(x)$ is increasing, it suffices to show that when $x=z_{1}^{*}, x-\theta_{\mathrm{H}}-\mu-\tilde{\alpha}_{\mathrm{H}}(x)$ is less than $q^{*}$. Note that at $x=z_{1}^{*}$, we have

$$
\begin{aligned}
x= & \theta_{\mathrm{H}}-\mu-\tilde{\alpha}_{\mathrm{H}}(x) \\
= & \theta_{\mathrm{L}}+\mu+\frac{1}{1+\gamma \sigma^{2}}+\Phi^{-1}\left(\frac{p-c+\frac{\rho}{1-\rho}\left(\theta_{\mathrm{H}}-\theta_{\mathrm{L}}\right)}{p+h}\right) \\
& -\theta_{\mathrm{H}}-\mu-\frac{1}{1+\gamma \sigma^{2}} \\
= & \theta_{\mathrm{L}}-\theta_{\mathrm{H}}+\Phi^{-1}\left(\frac{p-c+\frac{\rho}{1-\rho}\left(\theta_{\mathrm{H}}-\theta_{\mathrm{L}}\right)}{p+h}\right) .
\end{aligned}
$$

From Lemma 2 and conditions of part (1), this is less than $q^{*}$. Similarly, to verify that $z_{2}^{*}<q^{*}+\theta_{\mathrm{H}}+\mu+\frac{1}{1+\gamma \sigma^{2}}$, form the definition of $z_{2}^{*}$, it suffices to show that at $x=q^{*}+\theta_{\mathrm{H}}+\mu+\frac{1}{1+\gamma \sigma^{2}}, x-\theta_{\mathrm{H}}-\mu-\tilde{\alpha}_{\mathrm{H}}(x)$ is greater than $q^{*}$. It is known that

$$
\tilde{\alpha}_{\mathrm{H}}\left(q^{*}+\theta_{\mathrm{H}}+\mu+\frac{1}{1+\gamma \sigma^{2}}\right)<\frac{1}{1+\gamma \sigma^{2}} .
$$

Hence at $x=q^{*}+\theta_{\mathrm{H}}+\mu+\frac{1}{1+\gamma \sigma^{2}}$, we have

$$
x-\theta_{\mathrm{H}}-\mu-\tilde{\alpha}_{\mathrm{H}}(x)=q^{*}+\theta_{\mathrm{H}}+\mu+\frac{1}{1+\gamma \sigma^{2}}-\theta_{\mathrm{H}}-\mu
$$

Naval Research Logistics DOI 10.1002/nav 


$$
\begin{aligned}
& -\tilde{\alpha}_{\mathrm{H}}\left(q^{*}+\theta_{\mathrm{H}}+\mu+\frac{1}{1+\gamma \sigma^{2}}\right) \\
= & q^{*}+\frac{1}{1+\gamma \sigma^{2}}-\tilde{\alpha}_{\mathrm{H}}\left(q^{*}+\theta_{\mathrm{H}}+\mu+\frac{1}{1+\gamma \sigma^{2}}\right)>q^{*} .
\end{aligned}
$$

We first consider part (1), that is, the case where condition (a) or (b) holds. In this case, $\tilde{\alpha}_{\mathrm{H}}(x)$ and $\tilde{\alpha}_{\mathrm{L}}(x)$ cross twice, the first time at $z_{1}^{*}$, and the second time at $z_{3}^{*}$. If $x \in I_{1}, I_{2}$, or $I_{5}$, the optimal solution of (15) is the same as that without the $\alpha_{\mathrm{H}} \geq \alpha_{\mathrm{L}}$ constraint (see also Lemma 4). Hence, the optimal commission rates in these ranges are as given in the theorem. Now consider the case where $x \in I_{3}=\left[z_{1}^{*}, z_{2}^{*}\right)$, and examine optimization problem (15). As the optimal solution for the unconstrained optimization satisfies $\alpha_{\mathrm{L}}(x)>\alpha_{\mathrm{H}}(x)$, by Lemma 4 (b) the optimal solution for (15) has to be between the two optimal solutions. That is, we need to increase $\alpha_{\mathrm{H}}(x)$ but reduce $\alpha_{\mathrm{L}}(x)$ until they are equal. Note that at $\alpha_{\mathrm{H}}(x)=1 /\left(1+\gamma \sigma^{2}\right), G\left(q^{*} \vee\left(x-\theta_{\mathrm{H}}-\mu-\alpha_{\mathrm{H}}(x)\right)=G\left(q^{*}\right)\right.$ is flat as long as $x-\theta_{\mathrm{H}}-\mu-1 /\left(1+\gamma \sigma^{2}\right)<q^{*}$, and that when $\alpha_{\mathrm{H}}(x)$ increases, $G\left(q^{*} \vee\left(x-\theta_{\mathrm{H}}-\mu-\alpha_{\mathrm{H}}(x)\right)\right.$ remains constant. Hence, the optimal $\alpha_{\mathrm{H}}^{*}(x)=\alpha_{\mathrm{L}}^{*}(x)$ is the maximizer of

$$
\begin{aligned}
\rho\left[\alpha_{\mathrm{H}}-\frac{1+\gamma \sigma^{2}}{2} \alpha_{\mathrm{H}}^{2}\right]+(1-\rho)\left[\alpha_{\mathrm{L}}-\frac{\rho}{1-\rho} \alpha_{\mathrm{L}}\left(\theta_{\mathrm{H}}-\theta_{\mathrm{L}}\right)\right. \\
\left.\left.\quad-\frac{1+\gamma \sigma^{2}}{2} \alpha_{\mathrm{L}}^{2}-G\left(x-\theta_{\mathrm{L}}-\mu-\alpha_{\mathrm{L}}\right]\right)\right]
\end{aligned}
$$

Taking derivative we obtain the results given in part (iii). Therefore, it suffices to show that when $x \in I_{3}=\left[z_{1}^{*}, z_{2}^{*}\right)$, we have $x-\theta_{\mathrm{H}}-$ $\mu-1 /\left(1+\gamma \sigma^{2}\right)<q^{*}$. This was proved earlier, where we showed $z_{2}^{*}<q^{*}+\theta_{\mathrm{H}}+\mu+\frac{1}{1+\gamma \sigma^{2}}$. The result for the case where $x \in I_{4}$ follows a similar line of proof. Thus, it remains to prove part (2) of the theorem. The proof for this part follows Lemma 4 (a), since $\tilde{\alpha}_{\mathrm{H}}(x)$ and $\tilde{\alpha}_{\mathrm{L}}(x)$ do not cross in this case. That is, the objective function is separable in $\alpha_{\mathrm{H}}(x)$ and $\alpha_{\mathrm{L}}(x)$ (Lemma 3). Hence, by Lemma 4 (a), we can relax the constraint $\alpha_{\mathrm{L}}(x) \leq \alpha_{\mathrm{H}}(x)$ of (15), which results in the solutions given in parts (i)-(iii) of part (2). This completes the proof.

PROOF OF COROLLARY 1: Notice that when $\rho \geq(h+c+1) /(h+$ $\left.c+1+\left(\theta_{\mathrm{H}}-\theta_{\mathrm{L}}\right)\right), \lim _{x \rightarrow \infty} \tilde{\alpha}_{\mathrm{L}}(x)<0$, since $\Phi(\cdot)$ is a cdf function $\left(\tilde{\alpha}_{\mathrm{L}}(x)\right.$ is defined right before Theorem 1). Next, observe that from Theorem 1, for large enough inventory levels, $\alpha_{\mathrm{L}}^{*}(x)=\left(\tilde{\alpha}_{\mathrm{L}}(x)\right)^{+}$. Hence, $\lim _{x \rightarrow \infty} \alpha_{\mathrm{L}}^{*}(x)=0$. Moreover, since $\alpha_{\mathrm{L}}^{*}(x)$ is constrained to be nonnegative for all $x$, and is also increasing in $x$, it follows that $\alpha_{\mathrm{L}}^{*}(x)=0$ for all $x$. Furthermore, when $x \leq q^{*}+\theta_{\mathrm{L}}+\mu+\delta\left(1+\gamma \sigma^{2}\right)^{-1}$, from Theorem $1, \alpha_{\mathrm{L}}^{*}(x)=\delta\left(1+\gamma \sigma^{2}\right)^{-1}$. Hence, $\alpha_{\mathrm{L}}^{*}(x)=0$ if, and only if, $\delta \leq 0$, which is equivalent to $\rho \geq$ $1 /\left(1+\left(\theta_{\mathrm{H}}-\theta_{\mathrm{L}}\right)\right)$.

PROOF OF COROLLARY 2: To prove part (1), notice that from Theorem 1 and Lemma 1(a), the commission offered (as a function of the inventory level) to either $\mathrm{H}$ or L type agent is initially constant, followed by increasing functions. Furthermore, from Theorem 1, it follows that commissions are also decreasing in $\gamma$. The local concavity (i.e., concavity in each inventory interval) of the optimal commissions, follows directly by checking the second derivative of the optimal commissions given in Theorem 1.

PROOF OF COROLLARY 3: The proof follows directly from 3 (which shows that the effort induced by the firm is equal to the commission rate) and Corollary 2 part (1) (which shows that the commissions offered by the firm are increasing in her inventory level).

PROOF OF PROPOSITION 1: From (15), the only terms where $x$ appears is in $G\left(q^{*} \vee\left(x-\theta_{\mathrm{H}}-\mu-\alpha_{\mathrm{H}}\right)\right)$ and $G\left(q^{*} \vee\left(x-\theta_{\mathrm{L}}-\mu-\alpha_{\mathrm{L}}\right)\right)$, and both are jointly concave in $\left(x, \theta_{\mathrm{H}}, \alpha_{\mathrm{L}}\right)$. This shows that the objective of (15) is jointly concave in $\left(x, \alpha_{\mathrm{H}}, \alpha_{\mathrm{L}}\right)$. Since the constraint $\alpha_{\mathrm{H}} \geq \alpha_{\mathrm{L}} \geq 0$ defines a convex set, it follows from Proposition B-4 of page 525 of [23] that resulting optimal value function $V_{N}(x, \rho)$ is convex in $x$.

PROOF OF PROPOSITION 2: Using induction, assume $V_{n+1}(x, \rho)$ is concave in $x$. It follows that $W_{i}(y)$ (defined right before this proposition) is also concave. Since $q_{i}^{*}$ denotes the maximizer of $W_{i}(y)$, replacing $W_{i}(y)$ in the optimization problem (20) shows that it is optimal to set $y^{*}=$ $q_{i}^{*}+\theta_{i}+\mu_{n}+\alpha_{i}(x)$ or equivalently order $\left(q_{i}^{*}-x+\theta_{i}+\mu_{n}+\alpha_{i}(x)\right)^{+}$, where $(a)^{+}=\max \{a, 0\}$. This completes the proof.

PROOF OF COROLLARY 4: The results follows directly from Proposition 2 and Theorem 2 for Intervals $I_{1}$ and $I_{1}^{\prime \prime}$. Notice that on these intervals the optimal commission, $\alpha_{i}(x)$, is independent of $x$.

PROOF OF PROPOSITION 3: It is argued in the main body of the article that, for $i=\mathrm{L}, \mathrm{H}, W_{i}(x)$ is concave. Since $q_{i}^{*}$ is the maximizer of $W_{i}$, it follows that both $W_{\mathrm{H}}\left(q_{\mathrm{H}}^{*} \vee\left(x-\theta_{\mathrm{H}}-\mu_{n}-\alpha_{\mathrm{H}}\right)\right)$ and $W_{\mathrm{L}}\left(q_{\mathrm{L}}^{*} \vee\left(x-\theta_{\mathrm{L}}-\mu_{n}-\alpha_{\mathrm{L}}\right)\right)$ are decreasing concave in $x$. Rewrite (21) as

$$
\begin{aligned}
& V_{n}(x, \rho)-c x=\max _{\alpha_{\mathrm{H}}, \alpha_{\mathrm{L}}: \alpha_{\mathrm{H}} \geq \alpha_{\mathrm{L}} \geq 0}\left\{\frac{\ln U_{0}}{\gamma}+\bar{\theta}+\mu_{n}\right. \\
& +\rho\left[\alpha_{\mathrm{H}}-\frac{1+\gamma \sigma^{2}}{2} \alpha_{\mathrm{H}}^{2}+W_{\mathrm{H}}\left(q_{\mathrm{H}}^{*} \vee\left(x-\theta_{\mathrm{H}}-\mu_{n}-\alpha_{\mathrm{H}}\right)\right)\right] \\
& +(1-\rho)\left[\alpha_{\mathrm{L}}-\frac{\rho}{1-\rho} \alpha_{\mathrm{L}}\left(\theta_{\mathrm{H}}-\theta_{\mathrm{L}}\right)-\frac{1+\gamma \sigma^{2}}{2} \alpha_{\mathrm{L}}^{2}\right. \\
& \left.\left.+W_{\mathrm{L}}\left(q_{\mathrm{L}}^{*} \vee\left(x-\theta_{\mathrm{L}}-\mu_{n}-\alpha_{\mathrm{L}}\right)\right)\right]\right\} .
\end{aligned}
$$

Since, for each given feasible $\alpha_{\mathrm{H}}$ and $\alpha_{\mathrm{L}}$, the objective function in (37) is decreasing in $x$, it follows that $V_{n}(x)-c x$ is also decreasing in $x$. Concavity of $V_{n}(x)$ follows from the fact that the objective function is jointly concave in $\left(\alpha_{\mathrm{H}}, \alpha_{l}, x\right)$, and the feasible region is a convex set of $\left(\alpha_{\mathrm{H}}, \alpha_{\mathrm{L}}\right)$. Therefore, from Proposition B-4 of page 525 of [23] $V_{n}(x, \rho)-c x$, and hence $V_{n}(x, \rho)$, is concave in $x$.

PROOF OF LEMMA 5: (a) On the range of interest for $x, \alpha_{\mathrm{H}}(x)$ is the solution to (24). Taking derivative with respect to $x$ on both sides, we obtain

$$
-\left(1+\gamma \sigma_{n}^{2}\right) \alpha_{\mathrm{H}}^{\prime}(x)-W^{\prime \prime}\left(x-\theta_{\mathrm{H}}-\mu_{n}-\alpha_{\mathrm{H}}(x)\right)\left(1-\alpha_{\mathrm{H}}^{\prime}(x)\right)=0 .
$$

Solving for $\alpha_{\mathrm{H}}^{\prime}(x)$, and using the fact that $W(\cdot)$ is concave yields

$$
1 \geq \alpha_{\mathrm{H}}^{\prime}(x)=\frac{-W^{\prime \prime}\left(x-\theta_{\mathrm{H}}-\mu_{n}-\alpha_{\mathrm{H}}(x)\right)}{1+\gamma \sigma_{n}^{2}-W^{\prime \prime}\left(x-\theta_{\mathrm{H}}-\mu_{n}-\alpha_{\mathrm{H}}(x)\right)} \geq 0 .
$$

This shows that $\alpha_{\mathrm{H}}(x)$ and $x-\alpha_{\mathrm{H}}(x)$ are both increasing. Similar argument shows that $\alpha_{\mathrm{L}}(x)$ and $x-\alpha_{\mathrm{L}}(x)$ are increasing on $x \geq \max \left\{q_{\mathrm{H}}^{*}, q_{\mathrm{L}}^{*}\right\}+$ $\theta_{\mathrm{H}}+\mu_{n}+\frac{1}{1+\gamma \sigma_{n}^{2}}$ and completes the proof for part (a). To prove part (b), we first need the following simple result. Suppose $y$ is a vector with convex feasible set $C$ that is independent of $x$, and that $g(x, y)$ is decreasing in $x$ for any given $y$, then $\max _{y \in C} g(x, y)$ is also decreasing in $x$. Using this fact and (21) we can show by induction that

$$
\frac{\partial W(x, \rho)}{\partial x} \geq-\frac{h}{1-\alpha}
$$

for all $x$. Now write (24) as

$$
\left(1+\gamma \sigma^{2}\right) \alpha_{\mathrm{H}}(x)=1-W^{\prime}\left(x-\theta_{\mathrm{H}}-\alpha_{\mathrm{H}}(x)\right) .
$$


Since both $\alpha_{\mathrm{H}}(x)$ and $x-\alpha_{\mathrm{H}}(x)$ are increasing and that $W^{\prime}($.$) is decreas-$ ing, it follows that both sides of equation above have a limit. But the right-hand side is less than or equal to $1+h /(1-\alpha)$. Hence, $\alpha_{\mathrm{H}}(x)$ is increasing and converges to a finite number. Similar argument proves the result for $\alpha_{\mathrm{L}}(x)$.

PROOF OF THEOREM 2: The proof is similar to that of Theorem 1. First consider Case 1, and suppose $x \in I_{1}, I_{2}$ or $I_{5}$. The optimal solution of (21) in these intervals is the same as that without the constraint $\alpha_{\mathrm{H}} \geq \alpha_{\mathrm{L}}$ (see also Lemma 4). Hence, the optimal commissions in these ranges can be obtained from separately optimizing (22) and (23). Doing so, proves the result of parts (1), (2), and (5) of Case 1. When $x \in I_{3}$ or $x \in I_{4}$, the optimizers of (22) and (23) do not satisfy the constraint $\alpha_{\mathrm{H}} \geq \alpha_{\mathrm{L}}$. Hence, by Lemma 4 , the optimizers of (21) can be obtained by setting $\alpha_{\mathrm{H}}=\alpha_{\mathrm{L}}$. This yields the results provided in parts (3) and (4) of of Case 1. The proof for Case 2 (and also for the case where $\left.q_{\mathrm{H}}^{*}+\theta_{\mathrm{H}}+1 /\left(1+\gamma \sigma_{n}^{2}\right) \leq q_{\mathrm{L}}^{*}+\theta_{\mathrm{L}}+\delta /\left(1+\gamma \sigma_{n}^{2}\right)\right)$ follows the same line of argument by considering cases where separately optimizing (22) and (23) does or does not yield the optimizers of (21).

\section{ACKNOWLEDGMENTS}

The authors are grateful to the Editor-in-Chief, Prof. Awi Federgruen, the Associate Editor, and the anonymous referees for their constructive comments and suggestions, which helped to significantly improve the exposition of this article. The work of the second author was supported in part by NSF under grants CMMI-1131249 and CMMI-1362619.

\section{REFERENCES}

[1] P. Agrell and P. Bogetoft, Use of menu of incentive contracts. AG2:V4, Draft Report, Sumicsid, 2003, Available at: www.Sumicsid.com.

[2] A.A. Atkinson, Incentives, uncertainty and risk in the newsboy problem, Decis Sci 10 (1979), 341-353.

[3] A.K. Basu, K. Amiya, R. Lal, V. Srinivasan, and R. Staelin, Salesforce compensation plans: An agency theoretic perspective, Mark Sci 4 (1985), 267-291.

[4] P. Bhardwaj, Delegating pricing decisions, Mark Sci 20 (2001), 143-169.

[5] P. Bolton and M. Dewatripont, Contract theory, M.I.T. Press, Cambridge, MA, 2005.

[6] C.B. Cadsby, F. Song, and F. Tapon, Sorting and incentive effects of pay-for-performance: An experimental investigation, Acad Manag J 50 (2007), 387-405.

[7] C.J. Chang, Gas prices and automobile advertising expenditures in U.S. markets, Honors Thesis, Duke University, 2009.

[8] F. Chen, Sales-force incentives and inventory management, Manufact Serv Oper Manag 2 (2000), 186-202.

[9] F. Chen, Salesforce incentives, market information, and production/inventory planning, Manag Sci 51 (2005), 60-75.

[10] X. Chen and D. Simchi-Levi, Coordinating inventory control and pricing strategies with random demand and fixed ordering cost: The finite horizon case, Oper Res 52 (2004), 887-896.

[11] A.T. Coughlan, "Salesforce compensation: A review of MS/OR advances." in: J. Eliashberg and G. Lilien (Editors), Handbooks in Oper. Res. and Man. Sci.: Marketing, Vol. 5, Elsivier Science Publishers, North-Holland, Amsterdam, The Netherlands, 1993, pp. 611-651.

[12] A.T. Coughlan and S.K. Subrata, Salesforce compensation: Theory and managerial implications, Mark Sci 8 (1989), 324-342.
[13] Cybernet Data Systems, Inc., 10-Q: LKQ COPR. Item 2. Management's discussion and analysis of financial condition and results of operations. Available at: http://www.menafn.com/, Last access on 2011.

[14] T. Dai and K. Jerath, Salesforce compensation with inventory consideration, Manag Sci 59 (2013), 2490-2501.

[15] Y. Dai and X. Chao, Salesforce contract design and inventory planning with asymmetric risk-averse sales agents, Oper Res Lett 41 (2013), 86-91.

[16] N. DeHoratius and A. Raman, Store manager incentive design and retail performance: An exploratory investigation, Manufact Serv Oper Manag 9 (2007), 518-534.

[17] Y. Ding, R. Jia, and S. Tang, Dynamic principal agent model based on CMDP, Math Methods Oper Res 58 (2003), 149-157.

[18] M. Doepke and R.M. Townsend, Dynamic mechanism design with hidden income and hidden actions, J Econ Theory 126 (2006), 235-285.

[19] A. Federgruen and A. Heching, Combined pricing and inventory control under uncertainty, Oper Res 47 (1999), 454-475.

[20] X. Frexias, R. Guesnerie, and J. Tirole, Planning under incomplete information and the ratchet effect, Rev Econ Stud 52 (1985), 173-191.

[21] J. Gonik, Tie saleman's bonuses to forecasts, Harv Bus Rev 56 (1978), 116-123.

[22] H.S. Heese and J.M. Swaminathan, Inventory and sales effort management under unobservable lost sales, Eur J Oper Res 207 (2010), 1263-1268.

[23] D.P. Heyman and M.J. Sobel, Stochastic models in operations research, Vol. II, McGraw-Hill, New York, NY, 1984.

[24] W.J. Hopp, S.M.R. Iravani, and Z. Liu, The role of wholesalesalespersons and incentive plans in promoting supply chain performance. Working Paper, Department of Industrial Engineering and Management Science, Northwestern University, Evanston, IL, 2011.

[25] K. Jerath, S. Netssine, and Z.J. Zhang, Can we all get along? incentive contracts to bridge the marketing and operations divide. Working Paper, The Wharton School of Business, University of Pennsylvania, Philadelphia, PA, 2011.

[26] J.J. Laffont and D. Martimort, The theory of incentives: The principal-agent model, Princeton University Press, Princeton, 2001.

[27] J.J. Laffont and J. Tirole, Using cost observation to regulate firms, J Pol Econ 594 (1986), 614-641.

[28] J.J. Laffont and J. Tirole, Adverse selection and renegotiation in procurement, Rev Econ Stud 57 (1990), 597-625.

[29] R. Lal, Delegating pricing responsibility to the salesforce, Mark Sci 5 (1986), 159-168.

[30] M.A. Lariviere and E.L. Porteus, Stalking information: Bayesian inventory management with unobserved lost sales, Manag Sci 45 (1999), 346-363.

[31] A. Levenson, C. Zoghi, M. Gibbs, and G. Benson, Getting what you pay for: A case study of incentive pay optimization. Working Paper, Marshal School of Business, Univ. of S. Cal., 2010.

[32] H. Lutze and O. Ozer, Promised lead-time contracts under asymmetric information, Oper Res 56 (2008), 898-915.

[33] A. Mas-Colell, M.D. Whinston, and J.R. Green, Microeconomic theory, Oxford University Press, New York, 1995.

[34] B.K. Mishra and A. Prasad, Delegating pricing decisions in competitive markets with symmetric and asymmetric information, Mark Sci 24 (2005), 490-497.

[35] R. Myerson, Optimal coordination mechanisms in generalized principal-agent problems, J Math Econ 10 (1982), 67-81. 
[36] S. Oh and O. Ozer, Mechanism design for capacity planning under dynamic evolution of asymmetric demand forecasts. Working Paper, Stanford University, Stanford, CA, 2011.

[37] S.W. O'Leary-Kelly and B.E. Flores, The integration of manufacturing and marketing/sales decisions: Impact on organizational performance, J Oper Manag 20 (2002), 221-240.

[38] A. Pavan, I. Segal, and J. Toikka, Dynamic mechanism design: Incentive compatibility, profit maximization and information disclosure. Working paper, Northwestern University, 2011.

[39] E.L. Plambeck and S.A. Zenios, Performance-based incentives in a dynamic principal-agent model, Manufact Serv Oper Manag 2 (2000), 240-263.

[40] E.L. Plambeck and S.A. Zenios, Incentive efficient control of a make-to-stock production system, Oper Res 51 (2003), $371-386$.

[41] E. Porteus and S. Whang, On manufacturing/marketing incentives, Manag Sci 9 (1991), 1166-1181.
[42] C. Prendergastan, The provision of incentives in firms, J Econ Lit 36 (1999), 7-63.

[43] B. Salanie, The economics of contracts: A primer, MIT Press, Cambridge, MA, 1997.

[44] B.P. Shapiro, Can marketing and manufacturing coexist? Harv Bus Rev 55 (1977), 104-114.

[45] H. Zhang, M. Nagarajan, and G. Sosic, Dynamic supplier contracts under asymmetric inventory information, Oper Res 58 (2010), 1380-1397.

[46] H. Zhang and S.A. Zenios, A dynamic principal-agent model with hidden information: Sequential optimality through truthful state revelation, Oper Res 56 (2008), 371386.

[47] A. Zoltners P. Sinha, and G.A. Zoltners, The complete guide to accelerating sales force performance: How to get more sales from your sales force, AMACOM/American Management Association, 2001. 